\title{
Nuclear Reaction Rates in a Plasma
}

\author{
Lowell S. Brown \\ Department of Physics, University of Washington \\ Seattle, Washington 98195 \\ R. F. Sawyer \\ Department of Physics, University of California at Santa Barbara \\ Santa Barbara, California 93106
}




\begin{abstract}
The problem of determining the effects of the surrounding plasma on nuclear reaction rates in stars is formulated $a b$ initio, using the techniques of quantum statistical mechanics. Subject to the condition that the nuclear reactions ensue only at very close approach of the fusing ions and the condition that the reaction be slow, the authors derive a result that expresses the complete effects of Coulomb barrier penetration and of the influence of the surrounding plasma in terms of matrix elements of well defined operators. The corrections do not separate into the product of initial state and final state effects. When the energy release in the reaction is much greater than thermal energies, the corrections reduce, as expected, to evaluation of the equilibrium probability of one ion being very near to the position of another ion. We address the calculation of this probability in an approach that is based on perturbation theory in the couplings of the plasma particles to the two fusing particles, with the Coulomb force between the fusing particles treated nonperturbatively and interactions among the plasma particles treated in the one-loop approximation. We recapture standard screening effects, find a correction term that depends on the quantum mechanical nature of the plasma, and put an upper bound on the magnitude of the further correction terms for the case of a weakly coupled plasma. We find that possible "dynamical screening" effects that have been discussed in the literature are absent. The form of our results suggests that an approach that relies on numerical calculations of the correlation functions in a classical Coulomb gas, followed by construction of an effective two body potential and a quantum barrier penetration calculation, will miss physics that is as important as the physics that it includes.
\end{abstract}




\section{INTRODUCTION AND SUMMARY}

The theoretical determination of fusion rates in stars [For example, Clayton (1968)] divides into two steps: I. Finding the nuclear "S factor" for specific reactions, which, loosely speaking, characterizes what the rate of a specific nuclear reaction would be in the absence of the Coulomb repulsion between the fusing ions. II. Taking into account both the Coulomb repulsion between the fusing particles and the influence of the surrounding charged plasma [Reviewed in Ichimaru (1993)]. There is quite a clean division between these two steps under conditions such that the energy release in the fusion is very large compared with thermal energies, and such that the reaction rate is small. Both of these conditions are well satisfied in the case of nuclear reactions in the solar interior. In such cases, the result that we seek in step II is, to first approximation, the value at zero separation of the equilibrium densitydensity correlation function of the fusing ions.] We shall say nearly nothing further about step I in this paper, and turn directly to the barrier penetration and plasma aspect of the problem.

The history of this problem begins with the work of Gamow (1928), who calculated the Coulomb barrier effects in the limit in which the only Coulomb force taken into account is that between the two fusing bodies, which gives the answer for the limit of an extremely dilute plasma. The first important corrections to the Gamow rates are found in the results of Salpeter (1954) for the effects of Debye screening by the electrons and ions in the plasma.

\footnotetext{
${ }^{1}$ The fusion reaction itself, and the associated nuclear forces, change the behavior of wave functions at very short relative distances. But as long as the reaction rate is very small, either because of the Coulomb barrier penetration factor, or because the fusion interaction is weak, these changes are significant only over a very small volume, and the exterior plasma physics will have negligible effect on the space variation of wave function within the volume. The quantities that determine the influence of the plasma are then the correlation functions that would exist with the nuclear charges concentrated at points and the nuclear interactions turned off.
} 
These corrections, which will serve as the benchmark for comparison with further corrections, are given, at temperature $2=\beta^{-1}$, by

$$
\Gamma_{S}=\Gamma_{0} \exp \left\{\beta e_{1} e_{2} \kappa_{D}\right\}
$$

where $\Gamma_{0}$ is the rate in the absence of the plasma, $e_{1}, e_{2}$ are the charges of the fusing nuclei, and $\kappa_{D}$ is the Debye wave number. This wave number squared is the sum of those for each species in the plasma,

$$
\kappa_{D}^{2}=\sum_{s} \kappa_{D, s}^{2}
$$

Here we count each spin state of a particular kind of particle with a separate species index $s$. The Debye wave number is generally defined by the rate of exponential fall off of the chargecharge correlation function of the plasma. For a dilute plasma, the one-loop or random phase approximation gives, as is reviewed in Appendix A [Eq. (A32)],

$$
\kappa_{D, s}^{2}=4 \pi e_{s}^{2} \frac{\partial\left\langle n_{s}\right\rangle_{\beta}}{\partial \mu_{s}},
$$

where $\left\langle n_{s}\right\rangle_{\beta}$ is the average density of species $s$ which has charge $e_{s}=Z_{s} e$, and $\mu_{s}$ is the chemical potential of this species. The general form may be needed for the electron component in the plasma of a star in which the effects of Fermi statistics may be important, but for the ionic species, Boltzmann statistics suffices, in which case the Debye wave number assumes the familiar form

$$
\kappa_{D, s}^{2}=4 \pi e_{s}^{2} \beta\left\langle n_{s}\right\rangle_{\beta}
$$

The effects of the plasma on reaction rates in the sun, as estimated from the above formulae, are modest but important. For example, in the entire region from the center of the sun to the radius inside which one-half of the energy is generated, the screening effects give about a $5 \%$ increase in the rate of proton-proton fusion and a $20 \%$ increase in the rate

\footnotetext{
${ }^{2}$ We measure temperature in energy units so that the Boltzmann constant is unity, $k_{B}=1$.
} 
for the reaction $p+{ }^{7} \mathrm{Be} \rightarrow \gamma+{ }^{8} \mathrm{~B}$. In lower temperature main sequence stars, and in highly evolved stars, the corrections become even more important.

It is worthwhile exhibiting the physical origin of the Salpeter correction shown in Eq. (1.1), the leading correction in the dilute plasma case. A plasma shields a charge brought into it, with a point charge $q$ producing the Debye potential

$$
\phi(r)=\frac{q}{r} e^{-\kappa_{D} r} .
$$

Thus, assembling a charge distribution $\rho(\mathbf{r})$ in a plasma has an associated polarization energy - the energy to assemble the charge less the corresponding vacuum energy — given by

$$
\epsilon_{\rho}=\frac{1}{2} \iint(d \mathbf{r})\left(d \mathbf{r}^{\prime}\right) \rho(\mathbf{r})\left[\frac{e^{-\kappa_{D}\left|\mathbf{r}-\mathbf{r}^{\prime}\right|}}{\left|\mathbf{r}-\mathbf{r}^{\prime}\right|}-\frac{1}{\left|\mathbf{r}-\mathbf{r}^{\prime}\right|}\right] \rho\left(\mathbf{r}^{\prime}\right) .
$$

The limit for a point charge $q$ yields

$$
\epsilon_{q}=-\frac{1}{2} q^{2} \kappa_{D}
$$

The Boltzmann factor associated with this polarization energy alters the number density relation for the ions from that of a free gas to read

$$
\langle n\rangle_{\beta}=\lambda^{-3} e^{\beta \mu} e^{-\beta \epsilon_{q}},
$$

where $\lambda$ is the thermal wavelength defined by

$$
\lambda=\sqrt{\frac{2 \pi \beta}{M}},
$$

with $M$ the mass of the particle. A nuclear reaction rate depends upon the probability to find two particles at the same spot, or more precisely, upon the average of the product of the number densities of the two particles, $\left\langle n_{1}(0) n_{2}(0)\right\rangle$. With the two nuclear particles having charges $e_{1}$ and $e_{2}$, this is given by

\footnotetext{
${ }^{3}$ These estimates use the tables in Bahcall (1989), p. 90. The density, H/He ratio, and temperature all change considerably over this region, but the screening factor almost remains constant.
} 


$$
\left\langle n_{1}(0) n_{2}(0)\right\rangle_{\beta}=\lambda_{1}^{-3} \lambda_{2}^{-3} e^{\beta\left(\mu_{1}+\mu_{2}\right)} \exp \left\{-\beta \epsilon_{\left(e_{1}+e_{2}\right)}\right\}
$$

Thus, in view of the single-particle number density relation (1.8) and the form (1.7) of the polarization energy, we find that

$$
\left\langle n_{1}(0) n_{2}(0)\right\rangle_{\beta}=\left\langle n_{1}\right\rangle_{\beta}\left\langle n_{2}\right\rangle_{\beta} \exp \left\{\beta e_{1} e_{2} \kappa_{D}\right\}
$$

The exponential is the Salpeter correction in Eq. (1.1).

The method that we have just described is in the spirit of our work in this paper which makes use of grand canonical ensembles that entail chemical potentials. To make comparison with other treatments, we should note that the same result is obtained if one replaces the Coulomb potential between the fusing particles with a Debye screened potential of the form of Eq. (1.5). Since only distances that are short on the scale of the Debye length $\kappa_{D}^{-1}$ enter into the quantum tunneling, only the short-distance correction $\delta V=-e_{1} e_{2} \kappa_{D}$ to the Coulomb potential $e_{1} e_{2} / r$ need be retained. This is equivalent to an energy shift $E \rightarrow E+e_{1} e_{2} \kappa_{D}$ or a momentum alteration $\delta p=e_{1} e_{2} \kappa_{D} m / p$. Thus the Gamow tunneling factor $\exp \left\{-2 \pi e_{1} e_{2} m / p\right\}$ is corrected by the factor

$$
\exp \left\{e_{1} e_{2} \kappa_{D}\left(\frac{2 \pi e_{1} e_{2} m^{2}}{p^{3}}\right)\right\} .
$$

In the thermal average, as we review below [c.f. Eq. (3.15)], the relative momentum $p$ of the fusing particles is replaced by the most probable momentum $\bar{p}$, where

$$
\bar{p}^{3}=2 \pi e_{1} e_{2} m^{2} / \beta
$$

This replacement produces the Salpeter correction in Eq. (1.1).

The "weak screening" domain is defined as that in which the exponent in Eq. (1.1) is small compared to unity. This is also the condition under which the Debye formula gives a good approximation to the purely classical plasma correlations. Clearly, one way of going beyond the Salpeter correction and toward the domain of the "strongly coupled plasma" is to use a correlation function that is better than the one provided by the Debye formula. There is an extensive literature devoted to the computation of the classical correlation function for 
a plasma, and the determination of fusion rates therefrom [Salpeter and Van Horne (1969); Dewitt, Graboske and Cooper (1973); Graboske et al. (1973); Jancovici (1977)]. In these approaches one translates a classical correlation function, usually numerically determined, into a modification of the potential between the fusing particles, and then calculates the quantum mechanical barrier penetration factor, using this potential, in order to obtain the fusion rate from a Gamow factor appropriate to this potential. We call this the "basically classical" approach.

In this approach, there are conceptual problems raised by the division of the problem into a quantum mechanical and a classical part. The literature lacks any development that begins with a correct general expression for the rate, shows how a division into classical correlation plus quantum tunneling can be made as an approximation, and gives a system for finding the corrections to this approximation.t.

We shall develop a general formulation of the problem which is based on only one approximation - that the fusing nuclei in the plasma can themselves be described by MaxwellBoltzmann statistics. In practice, this is an excellent approximation as these particles are seldom so dense as to require quantum statistics. No approximation is required for the remainder of the plasma. This basic approximation enables us to disentangle the internal Coulomb corrections in the fusion process from the Coulomb interactions of the fusing nuclei

\footnotetext{
${ }^{4}$ The work of Alastuey and Jancovici (1978) does not completely meet these criteria for at least two technical reasons: 1) Dividing the system into subsystems of fusing particles and surrounding plasma, the authors treat the plasma completely classically, thereby missing the correction term that turns out to be numerically most significant for the weakly coupled case. 2) Their approximation scheme in which a numerically determined classical correlation function for the plasma can be applied to the quantum tunneling calculation for the fusing particles involves a zeroth order in which the fusing particles are frozen in an "average position" in space. As a result the effects of center of mass motion are irretrievably lost.
} 
with all the other particles in the plasma (including other nuclei that could fuse). We then employ this general formulation to obtain a straightforward approach to the problem, based on perturbation theory. Considered as a factor, the Salpeter correction differs from unity by a term of leading order $e^{3}$ and therefore should be calculable in a perturbation expansion. In describing our approach it will be convenient to identify two particular ions as the "fusing particles" and all of the rest of the ions and electrons in the surrounding medium as the "plasma particles". As we shall see explicitly later, the Salpeter correction can be derived as the leading correction from a perturbative treatment in which the Coulomb force between the fusing particles is treated exactly, the coupling between the fusing particles and the plasma particles is treated perturbatively, and the plasma itself is treated in one-loop order. The formulation does not divide the development into a quantum mechanical and a classical part.

We shall develop this approach in some detail, and address the corrections beyond the leading Salpeter term. We are still limited in application to a weak screening domain of density and temperature, but we are able to compare the correction terms with those that can be produced in the basically classical approach. If we had found rough agreement, then we could have argued that the basically classical approach is a reasonable way to approach the regions of stronger coupling. However we find the opposite, that the leading correction terms that emerge from the complete perturbative approach have a nature that is essentially different from those produced by the basically classical approach. Our results thus point to the need for a computational framework that does not divide into a classical and quantum part.

The new quantitative results applicable to the weak screening domain are:

1) It is frequently stated that the Salpeter result, or static screening, should be a good approximation only when the velocities of the plasma particles are greater than the velocities of the fusing particles, so that the latter see an average potential rather than a time dependent one [e.g. Johnson et al. (1992), Shaviv \& Shaviv (1996).] This condition is rather badly violated for the ionic component of the plasma. But we find, in the leading order 
of $e^{3}$, no "dynamical" modifications of the Salpeter result in the ionic component of the screening, in fact no dependence on the ionic masses at all. This conflicts with the results of Carrero, Schäfer, and Koonin (1988), who found such terms. These "dynamical" corrections to screening, if present, would produce moderate decreases in predicted fusion rates under solar conditions. In Appendix D we show how such terms, which never arise in our approach, are produced in a real time treatment. We also find the terms that cancel them.

2) There is one correction at the $e^{4}$ level that is numerically significant under solar conditions. The correction comes from a quantum mechanical term in the electronic part of the plasma response function, and is inaccessible to approaches based on a classical treatment of the plasma.

3) A bound is put on all remaining corrections at the $e^{4}$ level. This bound limits the contributions of such terms to around the $0.1 \%$ level, under solar conditions.

The sum of our new corrections turns out to be of marginal importance to the various solar neutrino puzzles [Bahcall (1989), (1995)]. Thus we conclude, as have others, that the resolutions to these puzzles will not be found in plasma physics.

\section{REACTION RATE THEORY}

\section{A. General Framework}

A nuclear reaction, which we schematically indicate by

$$
1+2 \rightarrow 3+4
$$

takes place over a very short distance in comparison with the particle separations in a

plasma. Hence, in the non-relativistic, second-quantized formalism which we employ, it can be described by an effective local Hamiltonian density

$$
\mathcal{H}(\mathbf{r}, t)=\mathcal{K}(\mathbf{r}, t)+\mathcal{K}^{\dagger}(\mathbf{r}, t)
$$

in which 


$$
\mathcal{K}(\mathbf{r}, t)=g e^{-i Q t} \psi_{4}^{\dagger}(\mathbf{r}, t) \psi_{3}^{\dagger}(\mathbf{r}, t) \psi_{2}(\mathbf{r}, t) \psi_{1}(\mathbf{r}, t),
$$

where $Q$ is the energy release of the reaction. The number density operator of a produced particle, say the particle labeled by 4 , is given by

$$
n(0)=\psi_{4}^{\dagger}(0) \psi_{4}(0)
$$

Neglecting the kinematical particle flow described by the divergence of the corresponding particle flux vector which vanishes in the ensemble average, the time rate of change of this density is given by

$$
\begin{aligned}
\dot{n}(0) & =-i \int(d \mathbf{r})[n(0), \mathcal{H}(\mathbf{r}, 0)] \\
& =-i\left\{\mathcal{K}(0)-\mathcal{K}^{\dagger}(0)\right\}
\end{aligned}
$$

These operators create and destroy particles, and hence their expectation vanishes in the unperturbed plasma ensemble which is diagonal in particle number. The reaction rate $\Gamma$ appears in the additional linear response to the action of the perturbation $\mathcal{H}$,

$$
\begin{aligned}
\Gamma & =-i \int_{-\infty}^{0} d t \int(d \mathbf{r})\langle[\dot{n}(0), \mathcal{H}(\mathbf{r}, t)]\rangle_{\beta} \\
& =-\int_{-\infty}^{0} d t \int(d \mathbf{r})\left\langle\left[\mathcal{K}(0)-\mathcal{K}^{\dagger}(0), \mathcal{K}(\mathbf{r}, t)+\mathcal{K}^{\dagger}(\mathbf{r}, t)\right]\right\rangle_{\beta},
\end{aligned}
$$

where $\langle\cdots\rangle_{\beta}$ denotes the grand canonical thermal average of the background plasma.

Since the operator $\mathcal{K}$ does not conserve particle number,

$$
\langle[\mathcal{K}(0), \mathcal{K}(\mathbf{r}, t)]\rangle_{\beta}=0=\left\langle\left[\mathcal{K}^{\dagger}(0), \mathcal{K}^{\dagger}(\mathbf{r}, t)\right]\right\rangle_{\beta} .
$$

Moreover, in virtue of the space-time translational invariance of the ensemble and the antisymmetry of the commutator,

$$
\left\langle\left[\mathcal{K}^{\dagger}(0), \mathcal{K}(\mathbf{r}, t)\right]\right\rangle_{\beta}=-\left\langle\left[\mathcal{K}(0), \mathcal{K}^{\dagger}(-\mathbf{r},-t)\right]\right\rangle_{\beta}
$$

Thus, changing the sign of the integration variables in this term, $(\mathbf{r}, t) \rightarrow(-\mathbf{r},-t)$, terms combine to produce an integral over all times, 


$$
\Gamma=-\int_{-\infty}^{+\infty} d t \int(d \mathbf{r})\left\langle\left[\mathcal{K}(0), \mathcal{K}^{\dagger}(\mathbf{r}, t)\right]\right\rangle_{\beta}
$$

The two terms which comprise the commutator which appear here correspond to the destruction of particle 4 via $3+4 \rightarrow 1+2$ as well as its creation by $1+2 \rightarrow 3+4$. We shall assume that initially there are no particles of type 3 or 4 present in the plasma - or at least that they are extremely dilute — so that the operator $\mathcal{K}^{\dagger}(\mathbf{r}, t)$ acting to the right on an initial state vanishes. Hence, in this case, the only case that we shall consider,

$$
\begin{aligned}
\Gamma & =\int_{-\infty}^{+\infty} d t \int(d \mathbf{r})\left\langle\mathcal{K}^{\dagger}(\mathbf{r}, t) \mathcal{K}(0)\right\rangle_{\beta} \\
& =g^{2} \int_{-\infty}^{+\infty} d t \int(d \mathbf{r}) e^{i Q t}\left\langle\psi_{1}^{\dagger}(\mathbf{r}, t) \psi_{2}^{\dagger}(\mathbf{r}, t) \psi_{3}(\mathbf{r}, t) \psi_{4}(\mathbf{r}, t) \psi_{4}^{\dagger}(0) \psi_{3}^{\dagger}(0) \psi_{2}(0) \psi_{1}(0)\right\rangle_{\beta} .
\end{aligned}
$$

The result (2.10) is equivalent to the 'golden rule' rate formula

$$
\Gamma=\sum_{F I} P(I)|\langle F=3,4 ; f|\mathcal{K}(0)| I=1,2 ; i\rangle|^{2}(2 \pi)^{4} \delta\left(E_{F}-E_{I}-Q\right) \delta\left(\mathbf{P}_{F}-\mathbf{P}_{I}\right)
$$

Here $|I=1,2 ; i\rangle$ describes the complete initial state of the system - the initial particles that take part in the reaction are labeled by 1,2 and the background plasma by $i$. The initial particles 1,2 are interacting with this background plasma, and so their separate energymomentum is not conserved. The initial states, however, can be chosen to be eigenstates of the total energy-momentum $E_{I}, \mathbf{P}_{I}$, and this we have done. The probability distribution for the initial states is denoted by $P(I)$. Similarly, the final state $\langle F=3,4 ; f|$ contains the produced particles 3,4 and describes the final plasma $f$ with the total energy-momentum of this state denoted by $E_{F}, \mathbf{P}_{F}$. The fusing particle coordinates and plasma coordinates are interlocked in a complex way in the states, making the golden rule formula somewhat ill adapted to proceeding further. In applications in which reaction energy release $Q$ is very large compared with thermal energies, or in a case in which the final particles 3 and 4 are both neutral, it is possible to express the rate in terms of a thermal correlation function of particles 1,2 in the initial state, but we can proceed quite some way from (2.10) without making these restrictions. 


\section{B. Free Gas}

We pause now in our general development to illustrate the nature of the result (2.10) which we have just derived by considering the limit in which the plasma is replaced by a free gas. For a free gas, the ensemble expectation value in Eq. (2.10) factorizes. Since the particles 3 and 4 are produced out of the vacuum, one factor is

$$
\left\langle 0\left|\psi_{3}(\mathbf{r}, t) \psi_{4}(\mathbf{r}, t) \psi_{4}^{\dagger}(0) \psi_{3}^{\dagger}(0)\right| 0\right\rangle=\int \frac{\left(d \mathbf{p}_{3}\right)}{(2 \pi)^{3}} \int \frac{\left(d \mathbf{p}_{4}\right)}{(2 \pi)^{3}} e^{i\left(\mathbf{p}_{3}+\mathbf{p}_{4}\right) \cdot \mathbf{r}-i\left(E_{3}+E_{4}\right) t},
$$

where

$$
E_{3}=\frac{\mathbf{p}_{3}^{2}}{2 M_{3}}, \quad E_{4}=\frac{\mathbf{p}_{4}^{2}}{2 M_{4}}
$$

The other factor is

$$
\left\langle\psi_{1}^{\dagger}(\mathbf{r}, t) \psi_{2}^{\dagger}(\mathbf{r}, t) \psi_{2}(0) \psi_{1}(0)\right\rangle_{\beta}=\int \frac{\left(d \mathbf{p}_{1}\right)}{(2 \pi)^{3}} n_{1}\left(\mathbf{p}_{1}\right) \int \frac{\left(d \mathbf{p}_{2}\right)}{(2 \pi)^{3}} n_{2}\left(\mathbf{p}_{2}\right) e^{-i\left(\mathbf{p}_{1}+\mathbf{p}_{2}\right) \cdot \mathbf{r}+i\left(E_{1}+E_{2}\right) t}
$$

where

$$
n_{1}\left(\mathbf{p}_{1}\right)=\frac{1}{e^{\beta\left(E_{1}-\mu_{1}\right)} \mp 1}
$$

and

$$
n_{2}\left(\mathbf{p}_{2}\right)=\frac{1}{e^{\beta\left(E_{2}-\mu_{2}\right)} \mp 1}
$$

are the Bose or Fermi distributions of the initial particles with chemical potentials $\mu_{1}$ and $\mu_{2}$ and energies

$$
E_{1}=\frac{\mathbf{p}_{1}^{2}}{2 M_{1}}, \quad E_{2}=\frac{\mathbf{p}_{2}^{2}}{2 M_{2}}
$$

With these results in hand, it easy to see that in the free gas limit, the rate formula (2.10) yields?

\footnotetext{
${ }^{5}$ Here we have neglected possible spin variables. Spin degeneracy is readily accounted for by including spin-weight factors in the density distributions $n_{1}\left(\mathbf{p}_{1}\right)$ and $n_{2}\left(\mathbf{p}_{2}\right)$.
} 


$$
\begin{aligned}
\Gamma_{0}=g^{2} \int \frac{\left(d \mathbf{p}_{4}\right)}{(2 \pi)^{3}} \int \frac{\left(d \mathbf{p}_{3}\right)}{(2 \pi)^{3}} \int \frac{\left(d \mathbf{p}_{2}\right)}{(2 \pi)^{3}} n_{2}\left(\mathbf{p}_{2}\right) \int \frac{\left(d \mathbf{p}_{1}\right)}{(2 \pi)^{3}} n_{1}\left(\mathbf{p}_{1}\right) \\
(2 \pi)^{3} \delta\left(\mathbf{p}_{4}+\mathbf{p}_{3}-\mathbf{p}_{2}-\mathbf{p}_{1}\right)(2 \pi) \delta\left(E_{4}+E_{3}-E_{2}-E_{1}-Q\right) .
\end{aligned}
$$

We use this free gas result to make several points. First of all, we note that it reproduces the exact nuclear rate

$$
\Gamma_{0}=\int \frac{\left(d \mathbf{p}_{2}\right)}{(2 \pi)^{3}} n_{2}\left(\mathbf{p}_{2}\right) \int \frac{\left(d \mathbf{p}_{1}\right)}{(2 \pi)^{3}} n_{1}\left(\mathbf{p}_{1}\right) \int d \sigma v
$$

if the coupling $g^{2}$ is taken inside the integration and replaced by the squared nuclear matrix element $|T|^{2}$. In practice, the effective Hamiltonian method produces the correct nuclear rate if the coupling $g^{2}$ is replaced by an appropriate average value of the squared matrix element $|T|^{2}$. Note that, as yet, the rate formula does not account for the Coulomb interactions between the reacting particles. Finally, we note that for most applications such as the plasma in ordinary stars, the initial particles 1 and 2 are in a dilute gas. In this case, the momentum-space densities may be replaced by the Maxwell-Boltzmann forms,

$$
n\left(\mathbf{p}_{1}\right)=e^{-\beta\left(E_{1}-\mu_{1}\right)}, \quad n\left(\mathbf{p}_{2}\right)=e^{-\beta\left(E_{2}-\mu_{2}\right)} .
$$

Expanding the Bose or Fermi denominators, we see that the correction to this leading approximation involves the very small factors $\exp \left\{\beta \mu_{1}\right\}$ and $\exp \left\{\beta \mu_{2}\right\}$. Since in the dilute gas limit

$$
\langle n\rangle_{\beta}=\int \frac{(d \mathbf{p})}{(2 \pi)^{3}} e^{-\beta(E-\mu)}=\lambda^{-3} e^{\beta \mu},
$$

we see that the corrections to the dilute limit are of order

$$
e^{\beta \mu} \approx\langle n\rangle_{\beta} \lambda^{3}
$$

which, for example, at the core of the sun is roughly of order $10^{-6}$. The corrections to the Maxwell-Boltzmann limit involve exchange effects of quantum identical particles. We turn now to exploit the dilute nature of the initial reacting particles so that they may be treated with classical statistics. 


\section{Plasma}

We shall work out the nuclear reaction rate accounting exactly for the Coulomb interactions between the reacting particles but taking advantage of their assumed dilute character so that we may neglect Bose or Fermi exchange effects. The other particles in the plasma, however, - such as electrons — need not be dilute, and they will not be restricted to obey classical statistics.

We shall assume that the produced particles, which are labeled by 3 and 4, are sparsely distributed in the plasma so that their initial state which appears in the rate formula (2.10) thermal expectation value $\langle\cdots\rangle_{\beta}$ can be represented by the vacuum state. Thus the evaluation of the rate involves the calculation of the vacuum expectation value in the subspace of the produced particles

$$
\mathcal{W}\left(\mathbf{r}_{3}, \mathbf{r}_{4}, t\right)=\left\langle 0_{3}, 0_{4}\left|\psi_{3}\left(\mathbf{r}_{3}, t\right) \psi_{4}\left(\mathbf{r}_{4}, t\right) \psi_{4}^{\dagger}(0) \psi_{3}^{\dagger}(0)\right| 0_{3}, 0_{4}\right\rangle
$$

in the limit $\mathbf{r}_{3}=\mathbf{r}=\mathbf{r}_{4}$. The Heisenberg equation of motion for the field operators gives

$$
\begin{aligned}
i \frac{\partial}{\partial t}\left\langle 0_{3}, 0_{4}\right| \psi_{3}\left(\mathbf{r}_{3}, t\right) \psi_{4}\left(\mathbf{r}_{4}, t\right)=\{ & \left.-\frac{\nabla_{3}^{2}}{2 M_{3}}-\frac{\nabla_{4}^{2}}{2 M_{4}}+\frac{e_{3} e_{4}}{\left|\mathbf{r}_{4}-\mathbf{r}_{3}\right|}\right\}\left\langle 0_{3}, 0_{4}\right| \psi_{3}\left(\mathbf{r}_{3}, t\right) \psi_{4}\left(\mathbf{r}_{4}, t\right) \\
& +\left\langle 0_{3}, 0_{4}\right| \psi_{3}\left(\mathbf{r}_{3}, t\right) \psi_{4}\left(\mathbf{r}_{4}, t\right)\left[e_{3} \phi\left(\mathbf{r}_{3}, t\right)+e_{4} \phi\left(\mathbf{r}_{4}, t\right)\right] .
\end{aligned}
$$

Here the operator $\phi(\mathbf{r}, t)$ is the potential produced by all the other particles in the plasma. It is determined by the operator $\rho\left(\mathbf{r}^{\prime}, t\right)$ which measures the charge density produced by all these particles,

$$
\phi(\mathbf{r}, t)=\int\left(d \mathbf{r}^{\prime}\right) \frac{1}{\left|\mathbf{r}-\mathbf{r}^{\prime}\right|} \rho\left(\mathbf{r}^{\prime}, t\right)
$$

In the absence of this potential, the bra vector in Eq. (2.24) defined by the field operators acting to the left on $\left\langle 0_{3}, 0_{4}\right|$ obeys

$$
i \frac{\partial}{\partial t}\left\langle\mathbf{r}_{3}, \mathbf{r}_{4}, t\right|=\left\langle\mathbf{r}_{3}, \mathbf{r}_{4}, t\right| H_{3,4}
$$

where, with ordinary quantum mechanical notation, 


$$
H_{3,4}=\frac{\mathbf{p}_{3}^{2}}{2 M_{3}}+\frac{\mathbf{p}_{4}^{2}}{2 M_{4}}+\frac{e_{3} e_{4}}{\left|\mathbf{r}_{3}-\mathbf{r}_{4}\right|}
$$

Thus,

$$
\left\langle\mathbf{r}_{3}^{\prime}=\mathbf{r}=\mathbf{r}_{4}^{\prime}, t\right|=\left\langle\mathbf{r}_{3}^{\prime}=\mathbf{r}=\mathbf{r}_{4}^{\prime}\right| e^{-i H_{3,4} t}
$$

The addition of the potential terms can be included by passing to an interaction picture with the time evolution operator

$$
W(t)=\left(\exp \left\{-i \int_{0}^{t} d t^{\prime}\left[e_{3} \phi\left(\mathbf{r}_{3}\left(t^{\prime}\right), t^{\prime}\right)+e_{4} \phi\left(\mathbf{r}_{4}\left(t^{\prime}\right), t^{\prime}\right)\right]\right\}\right)_{+}
$$

a time-ordered exponential in the real time $t$ with

$$
\mathbf{r}_{3 \text { or } 4}(t)=e^{i H_{3,4} t} \mathbf{r}_{3 \text { or } 4} e^{-i H_{3,4} t}
$$

Thus

$$
\left\langle 0_{3}, 0_{4}\right| \psi_{3}\left(\mathbf{r}_{3}, t\right) \psi_{4}\left(\mathbf{r}_{4}, t\right)=\left\langle\mathbf{r}_{3}, \mathbf{r}_{4}, t\right| W(t),
$$

and we conclude that

$$
\mathcal{W}\left(\mathbf{r}_{3}, \mathbf{r}_{4}, t\right)=\left\langle\mathbf{r}_{3}, \mathbf{r}_{4}, t|W(t)| \mathbf{r}_{3}=0=\mathbf{r}_{4}\right\rangle
$$

since this obeys the equation of motion (2.24) and the correct boundary conditions when $t \rightarrow 0$. The rate formula $(2.10)$ is now expressed as

$$
\Gamma=g^{2} \int_{-\infty}^{+\infty} d t \int(d \mathbf{r}) e^{i Q t}\left\langle\psi_{1}^{\dagger}(\mathbf{r}, t) \psi_{2}^{\dagger}(\mathbf{r}, t) \mathcal{W}(\mathbf{r}, \mathbf{r}, t) \psi_{2}(0) \psi_{1}(0)\right\rangle_{\beta}
$$

It should be noted that $\mathcal{W}(\mathbf{r}, \mathbf{r}, t)$ is an operator in the plasma state space.

The thermal expectation value which appears in Eq. (2.33) is defined by a sum over all states (except for the final particle states which have been accounted for), a trace weighted by the density operator

$$
\mathcal{P}=\frac{1}{Z} \exp \left\{-\beta\left[H-\sum_{a} \mu_{a} N_{a}\right]\right\}
$$


where $N_{a}$ are the number operators of the particles with chemical potential $\mu_{a}$, and $Z$ is the partition function

$$
Z=\operatorname{Tr} \exp \left\{-\beta\left[H-\sum_{a} \mu_{a} N_{a}\right]\right\}
$$

The density operator $\mathcal{P}$ defines the grand canonical ensemble that we employ. Since an exact treatment of the Coulomb interactions of the particular reacting particles requires an emphasis on the individual particle aspects, we are forced to employ the coordinate representation for these particles. Thus we use the coordinate representation for these particles in the trace that defines the average in the grand canonical ensemble and write, in a necessarily rather schematic way,

$$
\begin{aligned}
\left\langle\psi_{1}^{\dagger}(\mathbf{r}, t) \psi_{2}^{\dagger}(\mathbf{r}, t) \mathcal{W}(\mathbf{r}, \mathbf{r}, t) \psi_{2}(0) \psi_{1}(0)\right\rangle_{\beta}=\sum \frac{1}{N_{1} !} \frac{1}{N_{2} !} \int \cdots \\
\left\langle\mathbf{r}_{1}^{\prime} \cdots ; \mathbf{r}_{2}^{\prime} \cdots ; \cdots\left|\mathcal{P} \psi_{1}^{\dagger}(\mathbf{r}, t) \psi_{2}^{\dagger}(\mathbf{r}, t) \mathcal{W}(\mathbf{r}, \mathbf{r}, t) \psi_{2}(0) \psi_{1}(0)\right| \mathbf{r}_{1}^{\prime} \cdots ; \mathbf{r}_{2}^{\prime} \cdots ; \cdots\right\rangle .
\end{aligned}
$$

The sum is a sum over all particle numbers $N_{1}, N_{2}$ (which are now just numbers, not operators). The coordinates of all the particles that appear in the state vectors are to be integrated over all space. The abbreviated notation used to label these particles in a state vector such as

$$
\left|\mathbf{r}_{1}^{\prime} \cdots ; \mathbf{r}_{2}^{\prime} \cdots ; \cdots\right\rangle
$$

is as follows. The first set $\mathbf{r}_{1}^{\prime} \cdots$ denotes the coordinates of the $N_{1}$ particles of type 1 ; the second set $\mathbf{r}_{2}^{\prime} \cdots$ denotes the coordinates of the $N_{2}$ particles of type 2 ; the remaining ... stand for all the other particles. The sum defining the trace for these other particles may be done in any representation. In view of the symmetry in the integration over the initial reacting particles coordinates, the action of the destruction and creation operators is, effectively within this integration,

$$
\psi_{2}(0) \psi_{1}(0)\left|\mathbf{r}_{1}^{\prime} \cdots ; \mathbf{r}_{2}^{\prime} \cdots ; \cdots\right\rangle=|\cdots ; \cdots ; \cdots ;\rangle N_{1} N_{2} \delta\left(\mathbf{r}_{1}^{\prime}\right) \delta\left(\mathbf{r}_{2}^{\prime}\right)
$$

We now make the restriction that the initial reacting particles 1,2 are sufficiently dilute so that Boltzmann or classical statistics can be employed. In this approximation, exchange 
effects are neglected, and the particles that were destroyed by the field operators in Eq. (2.37) are identified with the particles destroyed when the remaining operators $\psi_{1}^{\dagger}(\mathbf{r}, t)$ and $\psi_{2}^{\dagger}(\mathbf{r}, t)$ act to the left. Accordingly, the ensemble average which appears in the rate formula (2.33) may be expressed as

$$
\begin{gathered}
\left\langle\psi_{1}^{\dagger}(\mathbf{r}, t) \psi_{2}^{\dagger}(\mathbf{r}, t) \mathcal{W}(\mathbf{r}, \mathbf{r}, t) \psi_{2}(0) \psi_{1}(0)\right\rangle_{\beta}=\sum \frac{1}{\left(N_{1}-1\right) !} \frac{1}{\left(N_{2}-1\right) !} \int \cdots \\
\left\langle\cdots ; \cdots ; \cdots\left|\left\langle\mathbf{r}_{1}^{\prime}=0=\mathbf{r}_{2}^{\prime}\left|\mathcal{P} \psi_{1}^{\dagger}(\mathbf{r}, t) \psi_{2}^{\dagger}(\mathbf{r}, t) \mathcal{W}(\mathbf{r}, \mathbf{r}, t)\right| 0_{1}, 0_{2}\right\rangle\right| \cdots ; \cdots ; \cdots\right\rangle \\
=\operatorname{Tr}\left\langle\mathbf{r}_{1}^{\prime}=0=\mathbf{r}_{2}^{\prime}\left|\mathcal{P} \psi_{1}^{\dagger}(\mathbf{r}, t) \psi_{2}^{\dagger}(\mathbf{r}, t)\right| 0_{1}, 0_{2}\right\rangle \mathcal{W}(\mathbf{r}, \mathbf{r}, t)
\end{gathered}
$$

The action of the creation operators on the vacuum state $\left|0_{1}, 0_{2}\right\rangle$ which appears here is essentially the adjoint of that appearing in Eq. (2.31), and so we have

$$
\psi_{1}^{\dagger}(\mathbf{r}, t) \psi_{2}^{\dagger}(\mathbf{r}, t)\left|0_{1}, 0_{2}\right\rangle=V(t)\left|\mathbf{r}_{1}^{\prime}=\mathbf{r}=\mathbf{r}_{2}^{\prime}, t\right\rangle
$$

Here the time dependence of the state is governed by the quantum-mechanical Hamiltonian

$$
H_{1,2}=\frac{\mathbf{p}_{1}^{2}}{2 M_{1}}+\frac{\mathbf{p}_{2}^{2}}{2 M_{2}}+\frac{e_{1} e_{2}}{\left|\mathbf{r}_{1}-\mathbf{r}_{2}\right|}
$$

with

$$
\left|\mathbf{r}_{1}^{\prime}=\mathbf{r}=\mathbf{r}_{2}^{\prime}, t\right\rangle=e^{i H_{1,2} t}\left|\mathbf{r}_{1}^{\prime}=\mathbf{r}=\mathbf{r}_{2}^{\prime}\right\rangle
$$

and

$$
V(t)=\left(\exp \left\{i \int_{0}^{t} d t^{\prime}\left[e_{1} \phi\left(\mathbf{r}_{1}\left(t^{\prime}\right), t^{\prime}\right)+e_{2} \phi\left(\mathbf{r}_{2}\left(t^{\prime}\right), t^{\prime}\right)\right]\right\}\right)_{-}
$$

is an anti-time-ordered exponential in the real time $t$ with

$$
\mathbf{r}_{1 \text { or } 2}(t)=e^{i H_{1,2} t} \mathbf{r}_{1 \text { or } 2} e^{-i H_{1,2} t}
$$

To deal with the action of the statistical density operator $\mathcal{P}$, we write the total Hamiltonian for the whole system which appears in $\mathcal{P}$ as

$$
H=H_{1,2}+e_{1} \phi\left(\mathbf{r}_{1}\right)+e_{2} \phi\left(\mathbf{r}_{2}\right)+H_{\mathrm{R}},
$$


where $\phi(\mathbf{r})$ is the potential that the remaining particles in the plasma produce at the point $\mathbf{r}$, and $H_{\mathrm{R}}$ is the Hamiltonian for the remaining particles in the plasma. In this way, we can make use of the interaction picture in imaginary time in writing

$$
\exp \{-\beta H\}=\exp \left\{-\beta\left(H_{1,2}+H_{\mathrm{R}}\right)\right\} U(\beta),
$$

in which

$$
U(\beta)=\left(\exp \left\{-\int_{0}^{\beta} d \tau\left[e_{1} \phi\left(\mathbf{r}_{1}(\tau), \tau\right)+e_{2} \phi\left(\mathbf{r}_{2}(\tau), \tau\right)\right]\right\}\right)_{+},
$$

is a time-ordered exponential in the imaginary time $\tau$ with

$$
\mathbf{r}_{1 \text { or } 2}(\tau)=e^{H_{1,2} \tau} \mathbf{r}_{1 \text { or } 2} e^{-H_{1,2} \tau} .
$$

The explicit $\tau$ dependence in the potential terms appears because the charge density of the background plasma moves in imaginary time under the action of $H_{\mathrm{R}}$. That is,

$$
\phi(\mathbf{r}, \tau)=\int\left(d \mathbf{r}^{\prime}\right) \frac{1}{\left|\mathbf{r}-\mathbf{r}^{\prime}\right|} \rho\left(\mathbf{r}^{\prime}, \tau\right),
$$

where

$$
\rho\left(\mathbf{r}^{\prime}, \tau\right)=e^{H_{\mathrm{R}} \tau} \rho\left(\mathbf{r}^{\prime}\right) e^{-H_{\mathrm{R}} \tau} .
$$

Using Eq. (2.45), we explicitly separate out the initial particles from the density operator to obtain

$$
\mathcal{P}=e^{\beta\left(\mu_{1}+\mu_{2}\right)} \overline{\mathcal{P}} e^{-\beta H_{1,2}} U(\beta),
$$

where $\overline{\mathcal{P}}$ is the density operator for the remaining particles. Hence with

$$
\left\langle\mathbf{r}_{1}^{\prime}=0=\mathbf{r}_{2}^{\prime}\right| e^{-\beta H_{1,2}}=\left\langle\mathbf{r}_{1}^{\prime}=0=\mathbf{r}_{2}^{\prime},-i \beta\right|,
$$

we have

$$
\begin{aligned}
\left\langle\mathbf{r}_{1}^{\prime}=0=\right. & \left.\mathbf{r}_{2}^{\prime}\left|\mathcal{P} \psi_{1}^{\dagger}(\mathbf{r}, t) \psi_{2}^{\dagger}(\mathbf{r}, t)\right| 0_{1}, 0_{2}\right\rangle= \\
& \exp \left\{\beta\left(\mu_{1}+\mu_{2}\right)\right\} \overline{\mathcal{P}}\left\langle\mathbf{r}_{1}^{\prime}=0=\mathbf{r}_{2}^{\prime},-i \beta|U(\beta) V(t)| \mathbf{r}_{1}^{\prime}=\mathbf{r}=\mathbf{r}_{2}^{\prime}, t\right\rangle
\end{aligned}
$$


Inserting this result in Eq. (2.38) produces a plasma thermal expectation value, and so Eq. (2.33) yields

$$
\begin{gathered}
\Gamma=g^{2} \exp \left\{\beta\left(\mu_{1}+\mu_{2}\right)\right\} \int_{-\infty}^{+\infty} d t \int(d \mathbf{r}) e^{i Q t} \\
\left\langle\left\langle\mathbf{r}_{1}^{\prime}=0=\mathbf{r}_{2}^{\prime},-i \beta|U(\beta) V(t)| \mathbf{r}_{1}^{\prime}=\mathbf{r}=\mathbf{r}_{2}^{\prime}, t\right\rangle\right. \\
\left.\left\langle\mathbf{r}_{3}^{\prime}=\mathbf{r}=\mathbf{r}_{4}^{\prime}, t|W(t)| \mathbf{r}_{3}^{\prime}=0=\mathbf{r}_{4}^{\prime}\right\rangle\right\rangle_{\beta} .
\end{gathered}
$$

This expression for the reaction rate is the major result of this paper. It separates out the plasma interactions with the particles undergoing the nuclear reaction from their internal Coulomb interactions. The plasma interactions have two different effects. One is to alter the thermal distribution describing the density of states of the initial two particles that enter into the reaction. This alteration is described by the operator $U(\beta)$. The other type of effect of the background plasma is to alter the dynamics of the motion of the particles undergoing the nuclear reaction. This is described by the operators $V(t)$ and $W(t)$.

Since our notation is rather condensed, it is worthwhile describing the meaning of this result in a little more detail. Spelling out the time dependence of the states expresses

$$
\begin{gathered}
\Gamma=g^{2} \exp \left\{\beta\left(\mu_{1}+\mu_{2}\right)\right\} \int_{-\infty}^{+\infty} d t \int(d \mathbf{r}) e^{i Q t} \\
\left\langle\left\langle\mathbf{r}_{1}^{\prime}=0=\mathbf{r}_{2}^{\prime}\left|e^{-\beta H_{1,2}} U(\beta) V(t) e^{i H_{1,2} t}\right| \mathbf{r}_{1}^{\prime}=\mathbf{r}=\mathbf{r}_{2}^{\prime}\right\rangle\right. \\
\left.\left\langle\mathbf{r}_{3}^{\prime}=\mathbf{r}=\mathbf{r}_{4}^{\prime}\left|e^{-i H_{3,4} t} W(t)\right| \mathbf{r}_{3}^{\prime}=0=\mathbf{r}_{4}^{\prime}\right\rangle\right\rangle_{\beta}
\end{gathered}
$$

Consider, for example, the second matrix element that appears within the thermal expectation value. Writing out the explicit expression (2.29) for the operator $W(t)$ ), this is the matrix element

$$
\begin{aligned}
& \left\langle\mathbf{r}_{3}^{\prime}=\mathbf{r}=\mathbf{r}_{4}^{\prime}, t|W(t)| \mathbf{r}_{3}^{\prime}=0=\mathbf{r}_{4}^{\prime}\right\rangle= \\
& \left\langle\mathbf{r}_{3}^{\prime}=\mathbf{r}=\mathbf{r}_{4}^{\prime}, t\left|\left(\exp \left\{-i \int_{0}^{t} d t^{\prime}\left[e_{3} \phi\left(\mathbf{r}_{3}\left(t^{\prime}\right), t^{\prime}\right)+e_{4} \phi\left(\mathbf{r}_{4}\left(t^{\prime}\right), t^{\prime}\right)\right]\right\}\right)_{+}\right| \mathbf{r}_{3}^{\prime}=0=\mathbf{r}_{4}^{\prime}\right\rangle .
\end{aligned}
$$

The potential $\phi\left(\mathbf{r}_{3}\left(t^{\prime}\right), t^{\prime}\right)$ is a field operator in the space of states that describe the thermal ensemble denoted by the overall expectation value $\langle\cdots\rangle_{\beta}$. This field operator is evaluated at the position $\mathbf{r}_{3}\left(t^{\prime}\right)$ which itself is an operator in the quantum-mechanical two-particle 
states of the final produced particles. The expectation value of Eq. (2.55), which is in the two-particle space of the produced particles, yields a functional of the field operators $\phi$ that depends parametrically upon the coordinate $\mathbf{r}$ and the time $t$. A similar functional of the field operator $\phi$ is produced by the other, initial particle, matrix element in Eq. (2.53). The reaction rate $\Gamma$ is then obtained by the space-time integral of the plasma, thermal expectation value of these functionals as shown in Eq. (2.53).

In solar fusion processes, the energy release $Q$ of the nuclear reaction is much larger than the temperature $T$. In such cases, the integrand in the time integration in Eq. (2.53) is dominated by the rapidly varying phase factor $\exp \{i Q t\}$ and the time dependence of the states $\left\langle\mathbf{r}_{3}^{\prime}=\mathbf{r}=\mathbf{r}_{4}^{\prime}, t\right|$. Including only this time variation, and setting $t=0$ elsewhere in Eq. (2.53), the time integration produces an energy-conserving $\delta$ function that sets the energy of the produced particles equal to the energy release $Q$. The time region that is important is of the order $1 / Q$, and the leading terms in the large $Q$ limit are obtained by placing $t=0$ in $V(t)$ and $W(t)$, which are thus replaced by unity, and by placing $t=0$ in the state $\left|\mathbf{r}_{1}^{\prime}=\mathbf{r}=\mathbf{r}_{2}^{\prime}, t\right\rangle$ as well, with corrections to this approximation roughly of order $T / Q$. We shall see this explicitly in later examples. Thus in the highly exothermic limit, the plasma dependence in the general rate formula (2.53) enters only in the quantity

$$
\begin{aligned}
& \left\langle\left\langle\mathbf{r}_{1}^{\prime}=\mathbf{r}=\mathbf{r}_{2}^{\prime},-i \beta|U(\beta)| \mathbf{r}_{1}^{\prime}=0=\mathbf{r}_{2}^{\prime}\right\rangle\right\rangle_{\beta}= \\
& \left\langle\mathbf{r}_{1}^{\prime}=0=\mathbf{r}_{2}^{\prime}\right| \exp \left\{-\beta H_{1,2}\right\} \\
& \left\langle\left(\exp \left\{-\int_{0}^{\beta} d \tau\left[e_{1} \phi\left(\mathbf{r}_{1}(\tau), \tau\right)+e_{2} \phi\left(\mathbf{r}_{2}(\tau), \tau\right)\right]\right\}\right)_{+}\right\rangle_{\beta}\left|\mathbf{r}_{1}^{\prime}=\mathbf{r}=\mathbf{r}_{2}^{\prime}\right\rangle .
\end{aligned}
$$

The spatial coordinate integration over $\mathbf{r}$ in Eq. (2.53) produces momentum $\delta$ functions that enforce the conservation of the total momentum in the nuclear reaction. As we shall also

\footnotetext{
${ }^{6}$ In the cases that we are considering, the thermal excitations in the plasma provide energy transfers of order $T$ which is much less than $Q$. However, in the interiors of stars that are more highly evolved than the sun, less exothermic and even endothermic reactions become important, and then the full complexity of the time dependence in Eq. (2.53) must be taken into account.
} 
describe later, the effect of this momentum conservation is to replace the ion masses in the plasma that have Coulomb interactions with the fusing nuclei by proper reduced masses that include the total mass of these nuclei. This takes into account the center-of-mass motion of the initial nuclear system through the plasma.

\section{RATE CALCULATIONS}

\section{A. Dilute Limit}

We first turn to illustrate the character of our basic result (2.53) with the limit where the plasma is very dilute. In this limit, the Coulomb interactions of the reacting particles with the background plasma may be neglected, and thus the operators $U(\beta), V(t)$, and $W(t)$ may be replaced by the identity operator. Thus in this dilute limit, the thermal expectation value is the trivial expectation value of the identity operator, and our result becomes simply

$$
\begin{aligned}
& \Gamma_{C}=g^{2} \exp \left\{\beta\left(\mu_{1}+\mu_{2}\right)\right\} \int_{-\infty}^{+\infty} d t \int(d \mathbf{r}) e^{i Q t} \\
& \quad\left\langle\mathbf{r}_{1}^{\prime}=0=\mathbf{r}_{2}^{\prime},-i \beta \mid \mathbf{r}_{1}^{\prime}=\mathbf{r}=\mathbf{r}_{2}^{\prime}, t\right\rangle\left\langle\mathbf{r}_{3}^{\prime}=\mathbf{r}=\mathbf{r}_{4}^{\prime}, t \mid \mathbf{r}_{3}^{\prime}=0=\mathbf{r}_{4}^{\prime}\right\rangle .
\end{aligned}
$$

To evaluate the transformation functions that appear here, we introduce complete sets of intermediate states which are eigenstates of the total initial or final momentum, $\mathbf{P}$ or $\mathbf{P}^{\prime}$, and of the initial or final relative momentum, $\mathbf{p}$ or $\mathbf{p}^{\prime}$. The relative momenta are the asymptotic momenta of the interacting scattering states. Thus

$$
\begin{aligned}
\left\langle\mathbf{r}_{1}^{\prime}=0=\mathbf{r}_{2}^{\prime},-i \beta \mid \mathbf{r}_{1}^{\prime}=\mathbf{r}=\mathbf{r}_{2}^{\prime}, t\right\rangle= & \int \frac{(d \mathbf{P})}{(2 \pi)^{3}} \exp \left\{-\frac{\mathbf{P}^{2}}{2 M}(\beta-i t)-i \mathbf{P} \cdot \mathbf{r}\right\} \\
& \int \frac{(d \mathbf{p})}{(2 \pi)^{3}} \exp \left\{-\frac{\mathbf{p}^{2}}{2 m}(\beta-i t)\right\}\left|\psi_{\mathbf{p}}^{(i)}(0)\right|^{2},
\end{aligned}
$$

and

$$
\begin{aligned}
\left\langle\mathbf{r}_{3}^{\prime}=\mathbf{r}=\mathbf{r}_{4}^{\prime}, t \mid \mathbf{r}_{3}^{\prime}=0=\mathbf{r}_{4}^{\prime}\right\rangle= & \int \frac{\left(d \mathbf{P}^{\prime}\right)}{(2 \pi)^{3}} \exp \left\{-i \frac{\mathbf{P}^{\prime 2}}{2 M} t+i \mathbf{P}^{\prime} \cdot \mathbf{r}\right\} \\
& \int \frac{\left(d \mathbf{p}^{\prime}\right)}{(2 \pi)^{3}} \exp \left\{-i \frac{\mathbf{p}^{\prime 2}}{2 m^{\prime}} t\right\}\left|\psi_{\mathbf{p}^{\prime}}^{(f)}(0)\right|^{2} .
\end{aligned}
$$


Here

$$
M=M_{1}+M_{2}=M_{3}+M_{4}
$$

is the common total mass of the initial and final states, and

$$
\frac{1}{m}=\frac{1}{M_{1}}+\frac{1}{M_{2}}, \quad \frac{1}{m^{\prime}}=\frac{1}{M_{3}}+\frac{1}{M_{4}}
$$

are the reduced masses of the initial and final states in the reaction. The squared amplitudes $\left|\psi_{\mathbf{p}}(0)\right|^{2}$ are the squared relative-motion Coulomb wave functions at the origin which have the form

$$
\left|\psi_{\mathbf{p}}(0)\right|^{2}=\frac{2 \pi \eta e^{-2 \pi \eta}}{1-e^{-2 \pi \eta}}
$$

with

$$
\eta=\frac{e_{1} e_{2} m}{|\mathbf{p}|}, \quad \eta^{\prime}=\frac{e_{3} e_{4} m^{\prime}}{\left|\mathbf{p}^{\prime}\right|}
$$

for the initial and final states.

The space-time integration in Eq. (3.1) now produces energy-momentum conserving $\delta$ functions, and we have

$$
\begin{gathered}
\Gamma_{C}=g^{2} \exp \left\{\beta\left(\mu_{1}+\mu_{2}\right)\right\} \int \frac{(d \mathbf{P})}{(2 \pi)^{3}} \exp \left\{-\beta \frac{\mathbf{P}^{2}}{2 M}\right\} \int \frac{(d \mathbf{p})}{(2 \pi)^{3}} \exp \left\{-\beta \frac{\mathbf{p}^{2}}{2 m}\right\}\left|\psi_{\mathbf{p}}^{(i)}(0)\right|^{2} \\
\int \frac{\left(d \mathbf{P}^{\prime}\right)}{(2 \pi)^{3}} \int \frac{\left(d \mathbf{p}^{\prime}\right)}{(2 \pi)^{3}}\left|\psi_{\mathbf{p}^{\prime}}^{(f)}(0)\right|^{2}(2 \pi)^{3} \delta\left(\mathbf{P}^{\prime}-\mathbf{P}\right)(2 \pi) \delta\left(\frac{\mathbf{p}^{\prime 2}}{2 m^{\prime}}-\frac{\mathbf{p}^{2}}{2 m}-Q\right) .
\end{gathered}
$$

Since $M m=M_{1} M_{2}$,

$$
\lambda_{1}^{-3} \lambda_{2}^{-3}=\left(\frac{M_{1}}{2 \pi \beta}\right)^{3 / 2}\left(\frac{M_{2}}{2 \pi \beta}\right)^{3 / 2}=\left(\frac{M}{2 \pi \beta}\right)^{3 / 2}\left(\frac{m}{2 \pi \beta}\right)^{3 / 2},
$$

and remembering the formula (2.22) for the number density, we see that

$$
\begin{gathered}
\Gamma_{C}=\left\langle n_{1}\right\rangle_{\beta}\left\langle n_{2}\right\rangle_{\beta} g^{2}\left(\frac{\beta}{2 \pi m}\right)^{3 / 2} \int(d \mathbf{p}) \exp \left\{-\beta \frac{\mathbf{p}^{2}}{2 m}\right\}\left|\psi_{\mathbf{p}}^{(i)}(0)\right|^{2} \\
\int \frac{\left(d \mathbf{p}^{\prime}\right)}{(2 \pi)^{3}}\left|\psi_{\mathbf{p}^{\prime}}^{(f)}(0)\right|^{2}(2 \pi) \delta\left(\frac{\mathbf{p}^{\prime 2}}{2 m^{\prime}}-\frac{\mathbf{p}^{2}}{2 m}-Q\right) .
\end{gathered}
$$


This is of the form

$$
\Gamma_{C}=\left\langle n_{1}\right\rangle_{\beta}\left\langle n_{2}\right\rangle_{\beta} \int\langle d \sigma v\rangle_{\beta},
$$

where now the differential cross section $d \sigma$ for the reaction includes the Coulomb corrections for the initial and final states.

Before turning to the plasma corrections, let us examine the scale - the size - of the variables that enter into the reaction rate calculation. Clearly, the common center-of-mass momentum for the initial and final states is governed by the Boltzmann factor. Thus, in order-of-magnitude,

$$
P=P^{\prime} \sim \sqrt{\frac{2 M}{\beta}} .
$$

The corresponding extent of the motion of the center of mass in imaginary time is of order

$$
\Delta R \sim(P / M) \beta \sim \sqrt{\frac{\beta}{M}} \sim \Lambda,
$$

where $\Lambda$ is the thermal wavelength of the center-of-mass motion. The magnitude of the initial relative momentum is determined by the balance of two factors, the Boltzmann factor which rapidly decreases as this momentum increases and the Coulomb barrier factor whose effect weakens as the relative momentum increases. In the usual applications, $2 \pi \eta=2 \pi e_{1} e_{2} \mathrm{~m} / \mathrm{p}$ is a moderately large number in the momentum region of relevance, and the value (3.6) of the Coulomb wave function at the origin is dominated by the leading exponential so that the distribution of initial relative momentum is peaked at the minimum value of the exponent in the factor

$$
\exp \left\{-\beta \frac{p^{2}}{2 m}-2 \pi \frac{e_{1} e_{2} m}{p}\right\},
$$

which is the point $p=\bar{p}$ given by

$$
\bar{p}^{3}=2 \pi e_{1} e_{2} m^{2} / \beta .
$$

At this point, the Coulomb parameter has the value 


$$
\bar{\eta}=\frac{e_{1} e_{2} m}{\bar{p}}=\left(\frac{e_{1}^{2} e_{2}^{2} m \beta}{2 \pi}\right)^{1 / 3}
$$

and the exponential displayed in Eq. (3.14) becomes $\exp \{-3 \pi \bar{\eta}\}$. Note that

$$
\lambda_{12}=\sqrt{\frac{2 \pi \beta}{m}}
$$

defines a thermal wavelength, while

$$
a_{12}=\frac{1}{e_{1} e_{2} m}
$$

defines the Bohr radius of the initial reacting system. In terms of these parameters,

$$
\bar{p}^{3}=\frac{(2 \pi)^{2}}{\lambda_{12}^{2} a_{12}}
$$

The corresponding kinetic energy $\bar{p}^{2} / 2 m$ is, in general, much less than the energy release $Q$ of the nuclear reaction. In these cases, the relative momentum of the produced particles is, in view of the energy-conserving $\delta$ function in the rate (3.10), of order

$$
p^{\prime} \sim \sqrt{2 m^{\prime} Q}
$$

which is much larger than the typical initial relative momentum $\bar{p}$.

The value (3.6) of the Coulomb wave function at the origin is given, to leading order, by a simple tunneling process. The classical turning point $r=r_{\max }$ which enters here, at the typical initial relative momentum $\bar{p}$, is determined by

$$
\frac{\bar{p}^{2}}{2 m}-\frac{e_{1} e_{2}}{r_{\max }}=0
$$

or

$$
r_{\max }=\frac{2 e_{1} e_{2} m}{\bar{p}^{2}}=\frac{2 \bar{\eta}}{\bar{p}}
$$

This sets the scale of the quantum motion in imaginary time for $r(\tau)$. Indeed, the tunneling amplitude for the initial two-particle state in a thermal ensemble at temperature $T=1 / \beta$ can be obtained as the steepest descent approximation to the path integral representation 
[Alastuey and Jancovici (1978)]. In this approximation, the exponential $\exp \{-3 \pi \bar{\eta}\}$ appears as the exponent of the classical action computed for a classical solution in imaginary time that has an orbit extending from $r=0$ to $r=r_{\max }$ back to $r=0$. This classical solution gives the leading approximation to the quantum motion.

\section{B. Plasma Corrections}

As we remarked in the discussion of the general rate formula (2.53), for highly exothermic reactions we may neglect the effects of the operators $V(t)$ and $W(t)$ so that the only plasma dependence is contained in the operator $U(\beta)$. The scale of spatial variations in the plasma is

controlled by the Debye length $\kappa_{D}^{-1}$ which is much larger than the estimates of the size of the coordinates $\mathbf{r}_{1 \text { or } 2}(\tau)$ which was just presented. Hence, we may, in the first approximation, set $\mathbf{r}=0$ in the potential operator $\phi(\mathbf{r}(\tau), \tau)$. As we shall soon see, the imaginary time dependence in $\phi(\mathbf{r}, \tau)$ may also be neglected in leading order so that we may approximate

$$
U(\beta)=\exp \left\{-\beta\left(e_{1}+e_{2}\right) \phi(0)\right\}
$$

Assuming that the plasma ensemble may be described by uncorrelated, Gaussian statistics, we now have

$$
\langle U(\beta)\rangle_{\beta}=\exp \left\{\beta^{2}\left(e_{1}+e_{2}\right)^{2}(1 / 2)\langle\phi(0) \phi(0)\rangle_{\beta}\right\}
$$

Using the potential correlation function obtained in Appendix A, Eq. (A39),

$$
\left\langle\phi(\mathbf{r}) \phi\left(\mathbf{r}^{\prime}\right)\right\rangle_{\beta}=\frac{1}{\beta\left|\mathbf{r}-\mathbf{r}^{\prime}\right|}\left[1-\exp \left\{-\kappa_{D}\left|\mathbf{r}-\mathbf{r}^{\prime}\right|\right\}\right]
$$

we have

$$
\langle U(\beta)\rangle_{\beta}=\exp \left\{(1 / 2) \beta\left(e_{1}+e_{2}\right)^{2} \kappa_{D}\right\}
$$

It should be remarked that the exponent that appears here is generally not a large number so that the Gaussian statistics assumption is not needed. As remarked in the Introduction, 
factors combine to form the plasma corrected number densities, and as shown in Appendix B (assuming that the result exponentiates)

$$
\left\langle n_{1 \text { or } 2}\right\rangle_{\beta}=\lambda_{1 \text { or } 2}^{-3} \exp \left\{\beta \mu_{1 \text { or } 2}\right\} \exp \left\{(1 / 2) \beta e_{1 \text { or } 2}^{2} \kappa_{D}\right\} .
$$

Therefore, the leading plasma correction is given by

$$
\Gamma_{S}=\exp \left\{\beta e_{1} e_{2} \kappa_{D}\right\} \Gamma_{C},
$$

where $\Gamma_{C}$ is the Coulomb corrected rate (3.10). And, as remarked in the Introduction, this is the Salpeter corrected rate.

We turn now to examine the size of the terms that we have neglected. We consider first the "dynamical" correction. Since the important time region is the small time interval $0<|t|<1 / Q$, in which the reacting particles move little, in the first approximation we may neglect both the spatial and time coordinates in the potential operator $\phi(\mathbf{r}, t)$. Hence, in leading order, the dynamical corrections involve

$$
V(t) W(t)=\exp \left\{i t\left(e_{1}+e_{2}\right) \phi(0)\right\} \exp \left\{-i t\left(e_{3}+e_{4}\right) \phi(0)\right\}=1,
$$

since charge is conserved in the reaction, $e_{1}+e_{2}=e_{3}+e_{4}$. To obtain the first "dynamical correction", we note that since short times are involved, the dominant term is that with the least number of time integrations. This term involves the interference of a correction from the "statistical" $U(\beta)$ and one of the "dynamical" $V(t)$ or $W(t)$ factors,

$$
\pm i \beta\left(e_{1}+e_{2}\right) e_{r} \int_{0}^{t} d t^{\prime}\left\langle\phi(0)\left[\phi\left(\mathbf{r}\left(t^{\prime}\right), t^{\prime}\right)-\phi(0)\right]\right\rangle_{\beta} .
$$

Here the \pm is + for the $V(t)$ factor of the initial particles 1,2 and - for the $W(t)$ factor of the final particles 3,4 . The charge of one of the reacting particles is denoted by $e_{r}$ and $\mathbf{r}\left(t^{\prime}\right)$ is its position operator. This correction is examined in Appendix $\mathrm{E}$ which provides the estimate

$$
\left(\beta e_{1} e_{2} \kappa_{D}\right)\left\{\frac{\hbar \omega_{p}}{Q} \sqrt{\frac{1}{\beta Q}}\right\}
$$


where $\omega_{p}^{2} \sim \kappa_{D}^{2} / \beta M$ defines the plasma frequency of a typical ion in the plasma. We have reverted to ordinary units in displaying this factor with Planck's constant $\hbar$ to show explicitly that it is of a quantum mechanical nature. The factor in braces is generally a very small correction indeed to the basic Salpeter correction $\left(\beta e_{1} e_{2} \kappa_{D}\right)$. For example, at the center of the sun, and for $Q=1 \mathrm{MeV}$, the factor in braces is about $10^{-6}$. Although one might expect that the contribution of the electrons in the plasma with their larger plasma frequency would dominate the dynamical correction, as shown in Appendix E, the leading contribution of the electrons cancels when summed over all the reacting particles.

We turn at last to estimate the error involved in the leading rate correction which comes from the "statistical" $U(\beta)$. (A related and simpler calculation of the single-particle number density is presented in Appendix B.) To make this estimate, we examine the initial particle expectation value of the dilute plasma limit

$$
\begin{aligned}
& \left\langle\left\langle\mathbf{r}_{1}^{\prime}=0=\mathbf{r}_{2}^{\prime},-i \beta|U(\beta)| \mathbf{r}_{1}^{\prime}=\mathbf{r}=\mathbf{r}_{2}^{\prime}, t\right\rangle\right\rangle_{\beta} \\
& \simeq\left\langle\mathbf{r}_{1}^{\prime}=0=\mathbf{r}_{2}^{\prime},-i \beta\right|\left\{1+\frac{1}{2} \int_{0}^{\beta} d \tau d \tau^{\prime}\right. \\
& \left.\left\langle\left(\left[e_{1} \phi\left(\mathbf{r}_{1}(\tau), \tau\right)+e_{2} \phi\left(\mathbf{r}_{2}(\tau), \tau\right)\right]\left[e_{1} \phi\left(\mathbf{r}_{1}\left(\tau^{\prime}\right), \tau^{\prime}\right)+e_{2} \phi\left(\mathbf{r}_{2}\left(\tau^{\prime}\right), \tau^{\prime}\right)\right]\right)_{+}\right\rangle_{\beta}\right\} \\
& \left|\mathbf{r}_{1}^{\prime}=\mathbf{r}=\mathbf{r}_{2}^{\prime}, t\right\rangle .
\end{aligned}
$$

The plasma expectation value that appears here may be written as a spatial Fourier transform

$$
\left\langle\left(\phi(\mathbf{r}, \tau) \phi\left(\mathbf{r}^{\prime}, \tau^{\prime}\right)\right)_{+}\right\rangle_{\beta}=\int \frac{(d \mathbf{k})}{(2 \pi)^{3}} e^{i \mathbf{k} \cdot\left(\mathbf{r}-\mathbf{r}^{\prime}\right)} G_{\beta}\left(k, \tau-\tau^{\prime}\right) .
$$

To discuss the initial particle expectation value, we use the center-of-mass and relative variables,

$$
\mathbf{R}=\left(M_{1} \mathbf{r}_{1}+M_{2} \mathbf{r}_{2}\right) / M, \quad \mathbf{P}=\mathbf{p}_{1}+\mathbf{p}_{2},
$$

and

$$
\check{\mathbf{r}}=\mathbf{r}_{1}-\mathbf{r}_{2}, \quad \mathbf{p}=\left(M_{2} \mathbf{p}_{1}-M_{1} \mathbf{p}_{2}\right) / M,
$$


where, as before, $M=M_{1}+M_{2}$ is the total mass of the reacting system. In terms of these variables this expectation value has the direct product form

$$
\begin{aligned}
& \left\langle\mathbf{r}_{1}^{\prime}=0=\mathbf{r}_{2}^{\prime},-i \beta \quad|\cdots| \mathbf{r}_{1}^{\prime}=\mathbf{r}=\mathbf{r}_{2}^{\prime}, t\right\rangle \\
& =\left\langle\mathbf{R}^{\prime}=0\right| \exp \left\{-\beta \frac{\mathbf{P}^{2}}{2 M}\right\}\left\langle\check{\mathbf{r}}^{\prime}=0\right| \exp \left\{-\beta H_{r}\right\} \cdots \\
& \exp \left\{i \frac{\mathbf{P}^{2}}{2 M} t\right\}\left|\mathbf{R}^{\prime}=\mathbf{r}\right\rangle \exp \left\{i H_{r} t\right\}\left|\check{\mathbf{r}}^{\prime}=0\right\rangle,
\end{aligned}
$$

in which

$$
H_{r}=\frac{\mathbf{p}^{2}}{2 m}+\frac{e_{1} e_{2}}{|\check{\mathbf{r}}|}
$$

is the Hamiltonian for the relative motion of the initial system with reduced mass $m$.

We shall first work out the effects of the center-of-mass motion. In terms of the centerof-mass and relative coordinates,

$$
\mathbf{r}_{1}=\mathbf{R}+M_{2} \check{\mathbf{r}} / M, \quad \mathbf{r}_{2}=\mathbf{R}-M_{1} \check{\mathbf{r}} / M .
$$

Thus, using the Fourier transform (3.33) in evaluating the correction (3.32), we see that we need to calculate the time ordered product

$$
\left(\exp \left\{i \mathbf{k} \cdot\left[\mathbf{R}(\tau)-\mathbf{R}\left(\tau^{\prime}\right)\right]\right\}\right)_{+}
$$

The center-of-mass coordinate undergoes free particle motion in imaginary time,

$$
\mathbf{R}(\tau)=\mathbf{R}-i \mathbf{P} \tau / M
$$

Using the canonical commutation relations to order the exponential,

$$
\begin{aligned}
\exp \{i \mathbf{k} \cdot \mathbf{R}+\mathbf{k} \cdot \mathbf{P} \tau / M\} & =\exp \left\{\mathbf{k}^{2} \tau / 2 M\right\} \exp \{i \mathbf{k} \cdot \mathbf{R}\} \exp \{\mathbf{k} \cdot \mathbf{P} \tau / M\} \\
& =\exp \left\{-\mathbf{k}^{2} \tau / 2 M\right\} \exp \{\mathbf{k} \cdot \mathbf{P} \tau / M\} \exp \{i \mathbf{k} \cdot \mathbf{R}\}
\end{aligned}
$$

we find that

$$
\left(\exp \left\{i \mathbf{k} \cdot\left[\mathbf{R}(\tau)-\mathbf{R}\left(\tau^{\prime}\right)\right]\right\}\right)_{+}=\exp \left\{-\mathbf{k}^{2}\left|\tau-\tau^{\prime}\right| / 2 M\right\} \exp \left\{\mathbf{k} \cdot \mathbf{P}\left(\tau-\tau^{\prime}\right) / M\right\}
$$


With this result in hand, we can compute explicitly the center-of-mass contribution to the initial particle expectation value needed for the correction (3.32). Introducing a complete set of intermediate state total momentum eigenstates, we now have

$$
\begin{array}{r}
\left\langle\mathbf{R}^{\prime}=0\left|\exp \left\{-\beta \frac{\mathbf{P}^{2}}{2 M}\right\}\left(\exp \left\{i \mathbf{k} \cdot\left[\mathbf{R}(\tau)-\mathbf{R}\left(\tau^{\prime}\right)\right]\right\}\right)_{+} \exp \left\{i \frac{\mathbf{P}^{2}}{2 M} t\right\}\right| \mathbf{R}^{\prime}=\mathbf{r}\right\rangle \\
=\exp \left\{-\mathbf{k}^{2}\left|\tau-\tau^{\prime}\right| / 2 M\right\} \int \frac{(d \mathbf{P})}{(2 \pi)^{3}} \exp \{-i \mathbf{P} \cdot \mathbf{r}\} \\
\exp \left\{-\frac{\mathbf{P}^{2}}{2 M}(\beta-i t)\right\} \exp \left\{\mathbf{k} \cdot \mathbf{P}\left(\tau-\tau^{\prime}\right) / M\right\} .
\end{array}
$$

The spatial $(d \mathbf{r})$ integration which appears in the rate formula $(2.53)$ produces a $\delta$ function which identifies the initial and final center-of-mass momenta $\mathbf{P}$ and $\mathbf{P}^{\prime}$. Thus the timedependent factor $\exp \left\{i \frac{\mathbf{P}^{2}}{2 M} t\right\}$ in Eq. (3.42) cancels the factor $\exp \left\{-i \frac{\mathbf{P}^{2}}{2 M} t\right\}$ which is contained in the final produced particle matrix element. Thus, effectively, we may delete all the time dependence associated with the center-of-mass motion, delete the $\mathbf{r}$ dependence, and remove the integration over the center-of-mass momentum $\mathbf{P}^{\prime}$ of the final produced particles. With this understood, we may complete the square in the $(d \mathbf{P})$ integration in Eq. (3.42) and get, effectively,

$$
\begin{gathered}
\left\langle\mathbf{R}^{\prime}=0\left|\exp \left\{-\beta \frac{\mathbf{P}^{2}}{2 M}\right\}\left(\exp \left\{i \mathbf{k} \cdot\left[\mathbf{R}(\tau)-\mathbf{R}\left(\tau^{\prime}\right)\right]\right\}\right)_{+} \exp \left\{i \frac{\mathbf{P}^{2}}{2 M} t\right\}\right| \mathbf{R}^{\prime}=\mathbf{r}\right\rangle \\
=\left(\frac{M}{2 \pi \beta}\right)^{3 / 2} C\left(k, \tau-\tau^{\prime}\right),
\end{gathered}
$$

in which

$$
C\left(k, \tau-\tau^{\prime}\right)=\exp \left\{-\frac{\beta \mathbf{k}^{2}}{2 M} f\left(\tau-\tau^{\prime}\right)\right\},
$$

where

$$
f\left(\tau-\tau^{\prime}\right)=\frac{\left|\tau-\tau^{\prime}\right|}{\beta}\left(1-\frac{\left|\tau-\tau^{\prime}\right|}{\beta}\right) .
$$

In an attempt to restrain notational proliferation, we define the normalized expectation value of an operator $X$ by

$$
(|X|)=\frac{\left\langle\check{\mathbf{r}}^{\prime}=0\left|\exp \left\{-\beta H_{r}\right\} X \exp \left\{i H_{r} t\right\}\right| \check{\mathbf{r}}^{\prime}=0\right\rangle}{\left\langle\check{\mathbf{r}}^{\prime}=0\left|\exp \left\{-\beta H_{r}\right\} \exp \left\{i H_{r} t\right\}\right| \check{\mathbf{r}}^{\prime}=0\right\rangle} .
$$


We should note that the real time dependence that appears here - the $t$ dependence makes a negligible contribution, and one can set $t=0$ here. This time dependence only gives rise to energy alterations on the order of particle thermal energies, which are negligible compared to the energy release of the nuclear reaction. Using this notation and the results that we have thus far obtained, Eq. (3.32) becomes, with the "effective" caveats noted above still in place,

$$
\begin{aligned}
& \left\langle\left\langle\mathbf{r}_{1}^{\prime}=0=\mathbf{r}_{2}^{\prime},-i \beta|U(\beta)| \mathbf{r}_{1}^{\prime}=\mathbf{r}=\mathbf{r}_{2}^{\prime}, t\right\rangle\right\rangle_{\beta} \\
& \simeq\left\langle\mathbf{r}_{1}^{\prime}=0=\mathbf{r}_{2}^{\prime},-i \beta \mid \mathbf{r}_{1}^{\prime}=\mathbf{r}=\mathbf{r}_{2}^{\prime}, t\right\rangle\left\{1+\frac{1}{2} \int_{0}^{\beta} d \tau d \tau^{\prime} \int \frac{(d \mathbf{k})}{(2 \pi)^{3}} G_{\beta}\left(k, \tau-\tau^{\prime}\right) C\left(k, \tau-\tau^{\prime}\right)\right. \\
& \left(\mid\left(e_{1}^{2} \exp \left\{i \mathbf{k} \cdot\left[\check{\mathbf{r}}(\tau)-\check{\mathbf{r}}\left(\tau^{\prime}\right)\right] M_{2} / M\right\}+e_{2}^{2} \exp \left\{-i \mathbf{k} \cdot\left[\check{\mathbf{r}}(\tau)-\check{\mathbf{r}}\left(\tau^{\prime}\right)\right] M_{1} / M\right\}\right.\right. \\
& \left.\left.\left.\quad+2 e_{1} e_{2} \exp \left\{i \mathbf{k} \cdot\left[\frac{M_{2}}{M} \check{\mathbf{r}}(\tau)+\frac{M_{1}}{M} \check{\mathbf{r}}\left(\tau^{\prime}\right)\right]\right\}\right)_{+} \mid\right)\right\} .
\end{aligned}
$$

To the order that we require, the potential correlation function $G_{\beta}\left(k, \tau-\tau^{\prime}\right)$ is given by the "sum of ring graphs" approximation, as discussed in Appendix A. To get the leading terms for dilute plasmas, a simplification can be made by subtracting the value at $k=0$ of the function that multiplies $G_{\beta}\left(k, \tau-\tau^{\prime}\right)$ in Eq. (3.47), and adding it back as a separate term. In the difference term we can replace the "ring sum" for $G_{\beta}\left(k, \tau-\tau^{\prime}\right)$ by the lowest order bubble. In this way we simplify the integration in a manner that avoids a potential infrared problem and captures all terms of order $e^{3}$ and $e^{4}$ in the final result for the rate. For the term in which the multiplying function is evaluated at $k=0$, we retain the complete $G_{\beta}\left(k, \tau-\tau^{\prime}\right)$ whose long-distance Debye screening removes the infrared divergence, and we encounter an expression evaluated in Eq. (B16) of Appendix B,

$$
\int_{0}^{\beta} d \tau d \tau^{\prime} \int \frac{(d \mathbf{k})}{(2 \pi)^{3}} G_{\beta}\left(k, \tau-\tau^{\prime}\right)=\beta \kappa_{D}+8 \int_{0}^{\beta} d \tau d \tau^{\prime} \int_{0}^{\infty} d k \frac{1}{k} \frac{d}{d k} \Pi^{(0)}\left(k, \tau-\tau^{\prime}\right) .
$$

Here the first term involving the Debye wave number $\kappa_{D}$, which is of order $e$ not $e^{2}$, isolates the long-distance contribution. The second term involves the first-order polarization function computed in Appendix A, whose dilute form reads

$$
\Pi^{(0)}\left(k, \tau-\tau^{\prime}\right) \simeq \sum_{s} e_{s}^{2}\left\langle n_{s}\right\rangle_{\beta}^{(0)} C_{s}\left(k, \tau-\tau^{\prime}\right)
$$


where

$$
C_{s}\left(k, \tau-\tau^{\prime}\right)=\exp \left\{-\frac{\beta k^{2}}{2 M_{s}} f\left(\tau-\tau^{\prime}\right)\right\}
$$

Having removed the long-distance contribution, we may now make the replacement

$$
G_{\beta}\left(k, \tau-\tau^{\prime}\right) \simeq\left(\frac{4 \pi}{k^{2}}\right)^{2} \Pi^{(0)}\left(k, \tau-\tau^{\prime}\right)
$$

in the remaining terms, which are of the form

$$
G_{\beta}\left(k, \tau-\tau^{\prime}\right)\left[C\left(k, \tau-\tau^{\prime}\right) e^{i \mathbf{k} \cdot \mathbf{r}}-1\right]
$$

The time ordering involves the operators $\check{\mathbf{r}}(\tau)$ and $\check{\mathbf{r}}\left(\tau^{\prime}\right)$, each of whose components commute amongst themselves. Hence the operators in the time-ordered product may be treated like ordinary numbers in intermediate calculations. Thus we now encounter a sum of the form

$$
\sum_{s} e_{s}^{2}\left\langle n_{s}\right\rangle_{\beta}^{(0)} \int_{0}^{\beta} d \tau d \tau^{\prime}
$$

of terms of the form

$$
\begin{aligned}
\int \frac{(d \mathbf{k})}{(2 \pi)^{3}}\left(\frac{4 \pi}{k^{2}}\right)^{2} \exp \left\{-\frac{\beta k^{2}}{2 M_{s}} f\left(\tau-\tau^{\prime}\right)\right\} \\
e_{a}^{2}\left[\exp \left\{-\frac{\beta k^{2}}{2 M} f\left(\tau-\tau^{\prime}\right)\right\} e^{i \mathbf{k} \cdot \mathbf{r}}-1\right] .
\end{aligned}
$$

To evaluate this expression, we represent

$$
\left(\frac{1}{k^{2}}\right)^{2}=\int_{0}^{\infty} s d s e^{-s k^{2}}
$$

and complete the square in the Gaussian wave number integrals to evaluate them and write the expression (3.54) as

$$
\sqrt{4 \pi} e_{a}^{2} \int_{0}^{\infty} s d s\left[\left(s+a_{s}\right)^{-3 / 2} \exp \left\{-\frac{\mathbf{r}^{2}}{4\left(s+a_{s}\right)}\right\}-\left(s+b_{s}\right)^{-3 / 2}\right],
$$

where

$$
a_{s}=\frac{\beta}{2 \bar{M}_{s}} f\left(\tau-\tau^{\prime}\right)
$$


with $\bar{M}_{s}$ the reduced mass defined by

$$
\frac{1}{\bar{M}_{s}}=\frac{1}{M}+\frac{1}{M_{s}}
$$

and

$$
b_{s}=\frac{\beta}{2 M_{s}} f\left(\tau-\tau^{\prime}\right)
$$

The potential infrared divergence is reflected in the large $s$ behavior of the integrand in Eq. (3.56) in which the separate terms do not give convergent integrals. The cancellation of the divergent pieces is exhibited by partial integration of the leading terms which reduces the expression in Eq. (3.56) to

$$
-\sqrt{\pi} e_{a}^{2} \int_{0}^{\infty} d s \frac{1}{\left(s+a_{s}\right)^{3 / 2}}\left[4 a_{s}+\frac{s}{s+a_{s}} \mathbf{r}^{2}\right] \exp \left\{-\frac{\mathbf{r}^{2}}{4\left(s+a_{s}\right)}\right\}+8 e_{a}^{2} \sqrt{\pi b_{s}} .
$$

It is easy to check that the last term here involving $\sqrt{b}$ gives contributions that precisely cancel the second term on the right-hand side of Eq. (3.48). Omitting this term and making a simple change of variables presents the result as

$$
-4 e_{a}^{2} \sqrt{\pi a_{s}} \int_{0}^{1} \frac{d u}{\sqrt{u}}\left[1+\frac{(1-u)}{4 a_{s}} \mathbf{r}^{2}\right] \exp \left\{-\frac{u \mathbf{r}^{2}}{4 a_{s}}\right\} .
$$

As is discussed in Appendix B, the contribution of the electrons in the plasma should be separated out because their mass is so small relative to the nuclear reacting particles and the nuclei in the plasma. The relatively small electron mass means that effectively $a_{s} \rightarrow \infty$ or that we may take $\mathbf{r}^{2}=0$ for their contribution in Eq. (3.60). Thus, for the electron contribution, the reacting particles may be taken to be at the same spatial position, and the calculation of their effect is the same as that for the number density calculation presented in Appendix $\mathrm{B}$, but for a particle of charge $e_{1}+e_{2}$. We evaluate the electronic part of the second term on the right-hand-side of Eq. (3.48) using the results of this Appendix, which include the effects of Fermi-Dirac statistics \, to obtain

\footnotetext{
${ }^{7}$ Note that the Debye wave number which appears here should be computed with the quantummechanical form (1.3) for the electrons presented in Appendix A [( $\mathrm{A32})]$ if the Fermi statistics for these particles is important.
} 


$$
\begin{aligned}
& \left\langle\left\langle\mathbf{r}_{1}^{\prime}=0=\mathbf{r}_{2}^{\prime},-i \beta|U(\beta)| \mathbf{r}_{1}^{\prime}=\mathbf{r}=\mathbf{r}_{2}^{\prime}, t\right\rangle\right\rangle_{\beta} \\
& \simeq\left\langle\mathbf{r}_{1}^{\prime}=0=\mathbf{r}_{2}^{\prime},-i \beta \mid \mathbf{r}_{1}^{\prime}=\mathbf{r}=\mathbf{r}_{2}^{\prime}, t\right\rangle \\
& \quad\left\{1+\frac{1}{2}\left(e_{1}+e_{2}\right)^{2} \beta \kappa_{D}-\frac{1}{8}\left(e_{1}+e_{2}\right)^{2} \beta \frac{2 e^{2} m_{e}}{e^{-\beta \mu_{e}}+1}-X\right\},
\end{aligned}
$$

in which

$$
\begin{aligned}
X & =\sum_{s \neq e} e_{s}^{2}\left\langle n_{s}\right\rangle_{\beta}^{(0)} \int_{0}^{\beta} d \tau d \tau^{\prime} \sqrt{4 \pi a_{s}} \int_{0}^{1} \frac{d u}{\sqrt{u}} \\
& \left(\mid\left(e_{1}^{2}\left[1+\frac{(1-u)}{4 a_{s}}\left[\check{\mathbf{r}}(\tau)-\check{\mathbf{r}}\left(\tau^{\prime}\right)\right]^{2}\left(M_{2} / M\right)^{2}\right] \exp \left\{-u \frac{\left[\check{\mathbf{r}}(\tau)-\check{\mathbf{r}}\left(\tau^{\prime}\right)\right]^{2}\left(M_{2} / M\right)^{2}}{4 a_{s}}\right\}\right.\right. \\
+ & e_{2}^{2}\left[1+\frac{(1-u)}{4 a_{s}}\left[\check{\mathbf{r}}(\tau)-\check{\mathbf{r}}\left(\tau^{\prime}\right)\right]^{2}\left(M_{1} / M\right)^{2}\right] \exp \left\{-u \frac{\left[\check{\mathbf{r}}(\tau)-\check{\mathbf{r}}\left(\tau^{\prime}\right)\right]^{2}\left(M_{1} / M\right)^{2}}{4 a_{s}}\right\} \\
+ & \left.\left.2 e_{1} e_{2}\left[1+\frac{(1-u)}{4 a_{s}}\left[\frac{M_{2}}{M} \check{\mathbf{r}}(\tau)+\frac{M_{1}}{M} \check{\mathbf{r}}\left(\tau^{\prime}\right)\right]^{2}\right] \exp \left\{-u \frac{\left[\frac{M_{2}}{M} \check{\mathbf{r}}(\tau)+\frac{M_{1}}{M} \check{\mathbf{r}}\left(\tau^{\prime}\right)\right]^{2}}{4 a_{s}}\right\}\right)_{+}\right) .
\end{aligned}
$$

As we shall soon see, the third term inside of the curly bracket in Eq. (3.62) is the most important correction, after the Salpeter correction, for the case of a weakly coupled plasma. It involves the quantum mechanics of the plasma in an essential way. This fact leads to a dilemma in considering how to approach the regime of lower temperature or higher density, a region in which the perturbation expansion is not valid. The standard approach to this domain is to use non-perturbative methods to treat the plasma classically, and then to use the extracted effective potentials in the tunneling calculation that determine the rates. But the fact that the leading perturbative correction to the Salpeter result requires a quantum treatment of the plasma argues that the quantum mechanics of the plasma will be central to any meaningful calculation of the strongly interacting case.

The correction $X$ due the ionic component of the plasma is quite small for a weakly coupled plasma, as a bound which we shall shortly derive proves. But before doing this, it is worth giving more detail to explain the remark of the previous paragraph that the character of this correction for the weakly coupled case brings into question the standard classical approach for dense plasmas. The matrix elements of the position operator $\check{\mathbf{r}}(\tau)$ are 
controlled by the magnitude of the classical turning point $r_{\max }$ discussed above [Eq. (3.22)]. Thus the exponents in Eq. (3.63) involve the parametric ratio $r_{\max }^{2} / a_{s}$. In terms of the thermal wave length

$$
\bar{\lambda}_{s}^{2}=\frac{2 \pi \beta}{\bar{M}_{s}},
$$

the denominator in this parametric ratio appears as

$$
a_{s}=\frac{\bar{\lambda}_{s}^{2}}{4 \pi} f\left(\tau, \tau^{\prime}\right)
$$

The thermal wave length $\bar{\lambda}_{s}$ is a quantum length — in ordinary units, it is of order Planck's constant $\hbar$. At the center of a typical star like the sun, this quantum-mechanical parametric ratio is about unity. In such cases, no small parameter appears in the ionic correction, no further approximations may be performed, and a full quantum-mechanical evaluation is required. It must be emphasized that the relevant ratio $r_{\max } / \bar{\lambda}_{s}$ entails the time-dependent, quantum-mechanical aspect of the plasma. A static, classical treatment of the plasma for such corrections is unphysical and gives incorrect results.

We now turn to bound the correction $X$ and then evaluate this bound approximately. The bound is easily obtained from the remark that the quantum expectation values entailed in the correction $X$ have path integral representations of the form

$$
\left\langle\check{\mathbf{r}}^{\prime}=0\left|\exp \left\{-\beta H_{r}\right\}\left(F\left(\check{\mathbf{r}}(\tau), \check{\mathbf{r}}\left(\tau^{\prime}\right)\right)\right)_{+}\right| \check{\mathbf{r}}^{\prime}=0\right\rangle=\int[d \mathbf{r}] e^{-S} F\left(\mathbf{r}(\tau), \mathbf{r}\left(\tau^{\prime}\right)\right),
$$

in which the paths start at $\mathbf{r}(0)=0$ and end at $\mathbf{r}(\beta)=0$. The action functional $S$ is the classical action continued to imaginary time. Since the action $S$ is real, the measure $[d \mathbf{r}] \exp \{-S\}$ is positive. Thus the expectation value (3.66) is bounded when the numerical function $F\left(\mathbf{r}(\tau), \mathbf{r}\left(\tau^{\prime}\right)\right)$ is bounded. Denoting an exponent which appears in Eq. (3.63) by $u g$, we note that the bounded combinations

$$
\left|(1-u g) e^{-u g}\right| \leq 1
$$

appears, giving the resulting parameter integral 


$$
\int_{0}^{1} \frac{d u}{\sqrt{u}}=2 .
$$

Following the evaluation of Eq. (B20) described in Appendix B, the imaginary time integrals for these terms involve

$$
\int_{0}^{\beta} d \tau d \tau^{\prime} \sqrt{4 \pi a_{s}}=\frac{\pi}{8} \beta^{2} \bar{\lambda}_{s}
$$

Thus, bounding the remaining terms with

$$
\left|\int_{0}^{1} \frac{d u}{\sqrt{u}} e^{-u g}\right| \leq \int_{0}^{\infty} \frac{d u}{\sqrt{u}} e^{-u g}=\sqrt{\frac{\pi}{g}}
$$

we obtain the bound

$$
\begin{aligned}
& |X| \leq \sum_{s \neq e} \beta \kappa_{D, s}^{2}\left\{\frac{1}{16}\left(e_{1}+e_{2}\right)^{2} \bar{\lambda}_{s}+\frac{1}{4 \beta^{2}} \int_{0}^{\beta} d \tau d \tau^{\prime}\right. \\
& \left.\left(\left|\left(\left(e_{1}^{2} \frac{M_{2}}{M}+e_{2}^{2} \frac{M_{1}}{M}\right)\left|\check{\mathbf{r}}(\tau)-\check{\mathbf{r}}\left(\tau^{\prime}\right)\right|+2 e_{1} e_{2}\left|\frac{M_{2}}{M} \check{\mathbf{r}}(\tau)+\frac{M_{1}}{M} \check{\mathbf{r}}\left(\tau^{\prime}\right)\right|\right)_{+}\right|\right)\right\},
\end{aligned}
$$

where

$$
\kappa_{D, s}^{2}=4 \pi \beta e_{s}^{2}\left\langle n_{s}\right\rangle_{\beta}^{(0)}
$$

is the contribution of the plasma species $s$ to the squared Debye wave length.

We should note that although the first term in the curly brackets in the bound (3.71) involves the quantum-mechanical nature of the plasma since it involves the quantummechanical wavelength $\bar{\lambda}_{s}$, the second set of terms refer only to the classical aspect of the plasma, albeit as measured by quantum expectation values of the reacting particles. This second set of terms is, in fact, just the classical plasma limit of the ionic correction (3.63). They are obtained from the result (3.63) if the limit $\bar{\lambda}_{s} \rightarrow 0$ is taken. This is the limit in which the plasma is treated classically, the formal $\hbar \rightarrow 0$ limit, or the limit in which the plasma particles are taken to have infinite mass (along with the total mass of the reacting pair). This limit is equivalent to taking $a_{s} \rightarrow 0$. In the limit, the exponentials completely damp out the $u$ integration except at the end point $u=0$. Hence, the $u$ integration may be extended to infinity. The terms which survive in the limit yield precisely this second set of 
terms in Eq. (3.71). The connection of this limit with a computation which starts out with a classical plasma is spelled out in some detail in Appendix C.

To estimate the size of the remaining quantum expectation values in Eq. (3.71), we note, as has been previously mentioned, that the basic quantum tunneling process is well described by the steepest descent approximation to the path integral. Thus we may estimate the size of these expectation values by replacing their quantum operators by the classical solution to the Coulomb problem in imaginary time which obeys the boundary conditions $\mathbf{r}(0)=0=\mathbf{r}(\beta) \cdot \mathrm{P}^{\beta}$ It is easy to verify that this solution to

$$
\frac{d^{2} \mathbf{r}(\tau)}{d \tau^{2}}=-\frac{e_{1} e_{2}}{r(\tau)^{3}} \mathbf{r}(\tau),
$$

which gives the stationary path, has the parametric representation

$$
\begin{gathered}
\tau=\frac{\beta}{2 \pi}(\xi-\sin \xi) \\
\mathbf{r}(\tau)=\frac{1}{2} r_{\max } \hat{\mathbf{k}}(1-\cos \xi),
\end{gathered}
$$

where $0 \leq \xi \leq 2 \pi, r_{\max }$ is the classical turning point given in Eq. (3.22), and $\hat{\mathbf{k}}$ is an arbitrary unit vector. The resulting integral

$$
\begin{aligned}
I & =\int_{0}^{\beta} d \tau d \tau^{\prime}\left|\mathbf{r}(\tau)-\mathbf{r}\left(\tau^{\prime}\right)\right| \\
& =\left(\frac{\beta}{2 \pi}\right)^{2} \frac{1}{2} r_{\max } \int_{0}^{2 \pi} d(\xi-\sin \xi) d\left(\xi^{\prime}-\sin \xi^{\prime}\right)\left|\cos \xi-\cos \xi^{\prime}\right|
\end{aligned}
$$

\footnotetext{
${ }^{8}$ The relative error to this classical evaluation is given by the first quantum correction which is of order $\Delta r^{2}\left(2 / r_{\max }\right)^{2}$, where $\Delta r^{2}$ is the average squared quantum fluctuation about the classical path. As an examination of the quadratic fluctuation correction to the path integral shows, this quantum fluctuation is of the same order as that for a harmonic oscillator with the same frequency, namely $\Delta r^{2} \simeq 1 /(m \omega)=\beta /(2 \pi m)$. Using Eq's. (3.22) and (3.16), we find that $\Delta r^{2}\left(2 / r_{\max }\right)^{2} \simeq 1 / \bar{\eta}$, which is negligible when the Coulomb parameter $\bar{\eta}$ is large. At the center of the sun, $\bar{\eta} \simeq 2$, and so our classical evaluation of the bound is a little rough.
} 
may be computed by partial integrations which lead to

$$
\frac{d}{d x} \frac{d}{d x^{\prime}}\left|x-x^{\prime}\right|=-2 \delta\left(x-x^{\prime}\right)
$$

Thus

$$
I=\frac{8 r_{\max } \beta^{2}}{3 \pi^{2}}
$$

The remaining integral is given by

$$
\begin{aligned}
J & =\int_{0}^{\beta} d \tau d \tau^{\prime}\left|\frac{M_{2}}{M} \mathbf{r}(\tau)+\frac{M_{1}}{M} \mathbf{r}\left(\tau^{\prime}\right)\right| \\
& =\beta \frac{M_{2}+M_{1}}{M} \frac{\beta}{2 \pi} \frac{1}{2} r_{\max } \int_{0}^{2 \pi} d \xi(1-\cos \xi)^{2} \\
& =\frac{3}{4} r_{\max } \beta^{2}
\end{aligned}
$$

Within this approximation we have

$$
|X| \leq \sum_{s \neq e} \beta \kappa_{D, s}^{2}\left\{\frac{1}{16}\left(e_{1}+e_{2}\right)^{2} \bar{\lambda}_{s}+r_{\max }\left[\frac{2}{3 \pi^{2}}\left(e_{1}^{2} \frac{M_{2}}{M}+e_{2}^{2} \frac{M_{1}}{M}\right)+\frac{3}{8} e_{1} e_{2}\right]\right\} .
$$

We return to our result (3.62) and omit the correction due to the ionic component of the plasma. Using the Bohr radius of the hydrogen atom $a_{0}=1 / e^{2} m_{e}$ and noting that the terms involving $e_{1}^{2}$ and $e_{2}^{2}$ just provide the corrected number density - chemical relation which is described in Appendix B, we find that the plasma corrected rate is given by

$$
\Gamma_{P}=\left\{1+\beta e_{1} e_{2}\left[\kappa_{D}-\frac{1}{2 a_{0}} \frac{1}{e^{-\beta \mu_{e}}+1}\right]\right\} \Gamma_{C}
$$

where $\Gamma_{C}$ is the rate in the absence of plasma corrections given in Eq. (3.10). In writing this result we have neglected the quantum effects described by the correction $X$ given in Eq. (3.63). For the example given by the center of the sun, $r_{\max } / \lambda \approx 1 / 2$, and $\kappa_{D} \lambda \approx 2 \times 10^{-2}$. Taking $e_{1}=e_{2}$, we find that $|X|<\beta e_{1} e_{2} \kappa_{D} \times 1 \times 10^{-2}$. That is, the quantum correction $X$ is less than $1 \%$ of the basic Salpeter correction. At the center of the sun, the electron correction that has been kept in the result (3.81) above, the second term in the square brackets, involves $1 / 2 a_{0} \approx \kappa_{D} / 4$ and $\exp \left\{\beta \mu_{e}\right\} \approx 0.3$. Thus it forms a correction of about $10 \%$ relative to the basic Salpeter correction. Moreover, it should be kept in mind that with 
$\exp \left\{\beta \mu_{e}\right\} \approx 0.3$, the correct quantum expression (1.3) for the electron contribution to the Debye wave number is reduced by about $10 \%$ relative to its classical value (1.4).

Since the last, electronic correction which appears in Eq. (3.81),

$$
\left[\kappa_{D}-\frac{1}{2 a_{0}} \frac{1}{e^{-\beta \mu_{e}}+1}\right]
$$

is the leading correction to the basic Salpeter result, we should describe its nature in a little more detail. In our formal counting of orders in the electric charge $e$, the Debye wave number $\kappa_{D}$ is of order $e$, while the new correction is of order $e^{2}$. The inverse Bohr radius $1 / a_{0}$ is proportional to one power of Planck's constant $\hbar$, and so the new correction explicitly involves the quantum-mechanical character of the plasma. The final factor with the electron's chemical potential represents the effect of Fermi-Dirac statistics. When the

electrons are sufficiently dilute $\left(\exp \left\{\beta \mu_{e}\right\} \ll 1\right)$ so that the Boltzmann limit can be used, the new correction may be written as

$$
\left[\kappa_{D}-\frac{1}{8} \kappa_{D, e}^{2} \lambda_{e}\right]
$$

Here $\kappa_{D, e}^{2}$ is the electronic contribution to the squared Debye wave length, and $\lambda_{e}$ is the electron's thermal wave length defined long ago in Eq. (1.9). The thermal wave length is again proportional to $\hbar$, and we again see that the new correction is a first quantum correction.

\section{CONCLUSIONS}

We have developed a concise expression [Eq. (2.53)] for the combined effects of the surrounding plasma and the Coulomb barriers on reaction rates in a completely ionized plasma. The expression is applicable to any case in which the actual reaction takes place at very small particle separations, and in which the reaction rate is slow, in a well defined sense. In general, there can be significant Coulomb and plasma effects in both initial and final states, and these effects are entangled in the results. For the cases of importance 
in solar physics, in which the release of energy in the reaction is very large compared to thermal energies, the correction reduces to one which involves a factor [Eq. (2.56)] that is a generalization of the probability that one fusing ion is at the position of the other. This generalization takes account of the effects of the center-of-mass motion of the initial fusing nuclei through the plasma.

We addressed the calculation of this quantity in an approach that is based on perturbation theory in the couplings of the plasma particles to the two (distinguished) particles that will undergo fusion. The Coulomb force between the fusing particles is treated nonperturbatively through use of the action-minimizing imaginary time paths for tunneling problems; alternatively our expressions could be expanded in Coulomb wave functions. The interactions among the plasma particles were treated in one-loop order. Expressing the result for the rate as a correction factor times the result in the absence of the plasma, our calculation captures all terms in the correction factor of order $e^{3}$ and $e^{4}$.

The results consisted of a recapitulation of the standard Salpeter results for the effects of the electron and ionic plasma particles, plus a single new analytical term coming from the quantum nature of the electron plasma, plus a rigorous bound on the remaining terms of our expression, which turn out to make negligible contributions under solar conditions. We have two conclusions that are significant to calculations of solar processes:

1) The quantum mechanical term in the dynamics of the electron plasma gives a reduction in the fusion rate of about 10 percent of the Salpeter enhancement term, under the conditions that prevail in the core of the sun.

2) There is no "dynamical screening" modification of the Salpeter result, to leading order in the dimensional parameters. There is no dependence on the masses of the plasma ions of the terms that are appreciable under solar conditions. In consequence, the fusion rates are somewhat greater than those calculated by Carrero et al. (1988). In Appendix D we 
demonstrate both how such terms arise in a real time approach and how they are cancelled.9

The nature of the remaining small correction terms in our results, and the formal structure of the results as well, argue against an approach to the strongly coupled problem in which numerical results for the classical plasma are turned into effective two body potentials, followed by the quantum tunneling calculation to obtain rates. In the weakly coupled domain, however, the result of our systematic perturbation theory provides support for the way in which screening calculations have been used in the literature, subject to the modifications mentioned above. In particular, the interparticle spacing does not have to be much smaller than the Debye length, even though this assumption goes into the derivation of the classical Debye potential. We reemphasize the fact that our treatment nowhere used this potential.

The results of section 2 are general, and could be the first step in developing approximations for the strongly coupled case. The perturbation development in section 3 is applicable only to the weak screening domain. In view of the smallness of the $e^{4}$ terms that we found, this fact seems to present no problems under solar conditions, but will probably not be useful for the interiors of much smaller main sequence stars or more highly evolved stars.

\footnotetext{
${ }^{9}$ As of the time of the writing of this paper it seems likely that the most critical calculation of rates in the sun, for the purpose of understanding the solar neutrino signal, will be the comparison of the $p+{ }^{7} \mathrm{Be} \rightarrow \gamma+{ }^{8} \mathrm{~B}$ rate with that of the rate of electron capture on ${ }^{7} \mathrm{Be}$ [Bahcall (1995), Hata and Langacker (1995)]. Modification (1) gives about a 2\% decrease and modification (2) about a $2 \%$ increase for the $p+{ }^{7}$ Be reaction. Thus our results have little impact on the fusion side of the comparison. However, it would be interesting to evaluate the plasma effects on the electron capture process using the methods of this paper.

${ }^{10}$ We thus take issue with the remarks of Dar and Shaviv (1996), who question the applicability of the standard screening lore under solar conditions, based on the fact that this condition on the interparticle spacing is not satisfied.
} 


\section{ACKNOWLEDGMENTS}

This project started at the Aspen Center for Physics. One of the authors (LSB) has had fruitful discussions on this topic with L. G. Yaffe. He would also like to thank R. S. Steinke for checking the manuscript. This work was supported, in part, by the U.S. Department of Energy under Grant No. DE-FG03-96ER40956.

\section{APPENDIX A: THERMODYNAMIC FIELD THEORY REVIEW}

We review and collect here some results of multiparticle, non-relativistic field theory at finite temperature that are used in our work.'미

The dependence in imaginary time of an arbitrary operator is given by

$$
X(\tau)=e^{H \tau} X e^{-H \tau}
$$

The thermal average of two operators is defined by

$$
\left\langle A(\tau) B\left(\tau^{\prime}\right)\right\rangle_{\beta}=Z^{-1} \operatorname{Tr} \exp \left\{-\beta\left[H-\sum_{a} \mu_{a} N_{a}\right]\right\} A(\tau) B\left(\tau^{\prime}\right)
$$

where

$$
Z=\operatorname{Tr} \exp \left\{-\beta\left[H-\sum_{a} \mu_{a} N_{a}\right]\right\}
$$

is the grand canonical partition function. Thus, if the operators commute with the particle number operators $N_{a}$, there is the cyclic symmetry

$$
\left\langle A(\tau) B\left(\tau^{\prime}\right)\right\rangle_{\beta}=\left\langle B\left(\tau^{\prime}\right) A(\tau-\beta)\right\rangle_{\beta},
$$

which follows from the cyclic symmetry of the trace and the time dependence given in Eq. (A1). Accordingly, the Green's function — the ordered product in imaginary time of two operators,

\footnotetext{
${ }^{11}$ The review here is close in spirit to discussion in Chapter 2 of Brown (1992), especially Problems 3 and 4 .
} 


$$
G\left(\tau-\tau^{\prime}\right)=\left\langle\left(A(\tau) B\left(\tau^{\prime}\right)\right)_{+}\right\rangle_{\beta}
$$

is periodic in imaginary time with period $\beta$, and it may be expanded in a Fourier series,

$$
G\left(\tau-\tau^{\prime}\right)=\frac{1}{\beta} \sum_{n} e^{-i \omega_{n}\left(\tau-\tau^{\prime}\right)} g\left(i \omega_{n}\right)
$$

where

$$
\omega_{n}=2 \pi n / \beta .
$$

Going over to real time $(\tau \rightarrow i t)$, with

$$
X(t)=e^{i H t} X e^{-i H t},
$$

we write the thermal average of the commutator as the Fourier integral

$$
\left\langle\left[A(t), B\left(t^{\prime}\right)\right]\right\rangle_{\beta}=\int_{-\infty}^{\infty} \frac{d \omega}{2 \pi} c(\omega) e^{-i \omega\left(t-t^{\prime}\right)} .
$$

Using the cyclic symmetry (A4), a short calculation shows that, in the imaginary time interval $0<\tau<\beta$,

$$
\langle A(\tau) B(0)\rangle_{\beta}=\int_{-\infty}^{\infty} \frac{d \omega}{2 \pi} \frac{c(\omega)}{1-e^{-\beta \omega}} e^{-\omega \tau}
$$

Therefore,

$$
\begin{aligned}
g\left(i \omega_{n}\right) & =\int_{0}^{\beta} d \tau\langle A(\tau) B(0)\rangle_{\beta} e^{i \omega_{n} \tau} \\
& =\int_{-\infty}^{\infty} \frac{d \omega}{2 \pi} \frac{c(\omega)}{\omega-i \omega_{n}} .
\end{aligned}
$$

To relate these results to the charge-density correlations that we need for our work, we note that first-order perturbation theory shows that the introduction of a small external potential $\delta \phi_{\text {ext }}(\mathbf{r}, t)$ induces an average charge density given by

$$
\langle\delta \rho(\mathbf{r}, t)\rangle_{\beta}=\int\left(d \mathbf{r}^{\prime}\right) \int d t^{\prime} R\left(\mathbf{r}-\mathbf{r}^{\prime}, t-t^{\prime}\right) \delta \phi_{\mathrm{ext}}\left(\mathbf{r}^{\prime}, t^{\prime}\right)
$$

where 


$$
\begin{aligned}
R\left(\mathbf{r}-\mathbf{r}^{\prime}, t-t^{\prime}\right) & =-i\left\langle\left[\rho(\mathbf{r}, t), \rho\left(\mathbf{r}^{\prime}, t^{\prime}\right)\right]\right\rangle_{\beta} \theta\left(t-t^{\prime}\right) \\
& =\int \frac{(d \mathbf{k})}{(2 \pi)^{3}} \int \frac{d \omega}{2 \pi} r(k, \omega) e^{i \mathbf{k} \cdot\left(\mathbf{r}-\mathbf{r}^{\prime}\right)-i \omega\left(t-t^{\prime}\right)} .
\end{aligned}
$$

The similar Fourier transform of the commutator function itself, without the factor $-i$ and without the step function $\theta\left(t-t^{\prime}\right)$, defines a weight which we shall denote by $c(k, \omega)$. Since this commutator function is odd under the interchange $t \leftrightarrow t^{\prime}, c(k,-\omega)=-c(k, \omega)$ is odd in $\omega$. Since complex conjugation of the expectation value the commutator of the Hermitian fields $\rho$ is equivalent to this interchange, which is compensated by the complex conjugation of $\exp \left\{-i \omega\left(t-t^{\prime}\right)\right\}, c(k, \omega)^{*}=c(k, \omega)$ is a real function. The relationship between the Fourier transform of the retarded commutator function which appears in Eq. (A13) and the Fourier transform $c(k, \omega)$ of the commutator function itself is given by the dispersion relation

$$
r(k, \omega)=\int_{-\infty}^{\infty} \frac{d \omega^{\prime}}{2 \pi} \frac{c\left(k, \omega^{\prime}\right)}{\omega-\omega^{\prime}-i \epsilon},
$$

as one can easily prove by directly Fourier transforming over only positive time differences the Fourier representation of the commutator function. In view of the general relation (A11) between the commutator and Green's functions, we see that the charge-density correlator in imaginary time,

$$
\left\langle\left(\rho(\mathbf{r}, \tau) \rho\left(\mathbf{r}^{\prime}, \tau^{\prime}\right)\right)_{+}\right\rangle_{\beta}=\frac{1}{\beta} \sum_{n} e^{-i \omega_{n}\left(\tau-\tau^{\prime}\right)} \int \frac{(d \mathbf{k})}{(2 \pi)^{3}} g\left(k, i \omega_{n}\right) e^{i \mathbf{k} \cdot\left(\mathbf{r}-\mathbf{r}^{\prime}\right)},
$$

has a weight given by the analytic continuation

$$
g\left(k, i \omega_{n}\right)=-r\left(k, i \omega_{n}\right)
$$

The induced charge - perturbing potential relation in Fourier space,

$$
\begin{aligned}
\langle\delta \rho(\mathbf{k}, \omega)\rangle_{\beta} & =r(k, \omega) \delta \phi_{\mathrm{ext}}(\mathbf{k}, \omega) \\
& =\left[\frac{1}{\epsilon(k, \omega)}-1\right] \frac{k^{2}}{4 \pi} \delta \phi_{\mathrm{ext}}(\mathbf{k}, \omega),
\end{aligned}
$$

defines the dielectric function $\epsilon(k, \omega)$. Hence,

$$
g\left(k, i \omega_{n}\right)=\frac{k^{2}}{4 \pi}\left[1-\frac{1}{\epsilon\left(k, i \omega_{n}\right)}\right] .
$$


It is worthwhile noting that, reverting to ordinary units and restoring Planck's constant $\hbar, \omega_{n}=2 \pi n / \hbar \beta$. Hence in the classical limit $\hbar \rightarrow 0$, which is equivalent to the high temperature limit $\beta \rightarrow 0, \omega_{n} \rightarrow \infty$ if $n \neq 0$ and only the $n=0$ term contributes to the Fourier sum defining the correlation function. Thus in this limit, the correlation function becomes independent of the imaginary times, with

$$
\left\langle\rho(\mathbf{r}) \rho\left(\mathbf{r}^{\prime}\right)\right\rangle_{\beta} \simeq \frac{1}{\beta} \int \frac{(d \mathbf{k})}{(2 \pi)^{3}} \frac{k^{2}}{4 \pi}\left[1-\frac{1}{\epsilon(k, 0)}\right] e^{i \mathbf{k} \cdot\left(\mathbf{r}-\mathbf{r}^{\prime}\right)} .
$$

The dielectric function is related to the irreducible polarization function by

$$
\epsilon(k, \omega)=1+\frac{4 \pi}{k^{2}} \Pi(k, \omega)
$$

which gives

$$
g\left(k, i \omega_{n}\right)=\frac{\Pi\left(k, i \omega_{n}\right)}{1+\frac{4 \pi}{k^{2}} \Pi\left(k, i \omega_{n}\right)} .
$$

The "sum of ring graphs" approximation is obtained by approximating the exact polarization function which appears here by its lowest-order, one-loop form $\Pi^{(0)}$. This function is obtained by neglecting the denominator correction above and setting

$$
\Pi^{(0)}\left(\mathbf{r}-\mathbf{r}^{\prime}, \tau-\tau^{\prime}\right)=\left\langle\left(\rho(\mathbf{r}, \tau) \rho\left(\mathbf{r}^{\prime}, \tau^{\prime}\right)\right)_{+}\right\rangle_{\beta}^{(0)}
$$

where the superscript on the expectation value indicates that it is computed for free fields. The charge density operator which appears here has the field expression

$$
\rho(\mathbf{r}, \tau)=\sum_{s} e_{s} \psi_{s}^{\dagger}(\mathbf{r}, \tau) \psi_{s}(\mathbf{r}, \tau)
$$

where the sum runs over all the particle species with charge $e_{s}$ (and over all spin components). In the free field approximation,

$$
\left\langle\left(\rho(\mathbf{r}, \tau) \rho\left(\mathbf{r}^{\prime}, \tau^{\prime}\right)\right)_{+}\right\rangle_{\beta}^{(0)}=\sum_{s} e_{s}^{2}\left\langle\left(\psi_{s}(\mathbf{r}, \tau) \psi_{s}^{\dagger}\left(\mathbf{r}^{\prime}, \tau^{\prime}\right)\right)_{+}\right\rangle_{\beta}^{(0)}\left\langle\left(\psi_{s}^{\dagger}(\mathbf{r}, \tau) \psi_{s}\left(\mathbf{r}^{\prime}, \tau^{\prime}\right)\right)_{+}\right\rangle_{\beta}^{(0)}
$$

since the total charge vanishes and with it the expectation value of a single charge operator.

The field expectation values that appear here are obtained from the generic forms 


$$
\left\langle\psi(\mathbf{r}, \tau) \psi^{\dagger}\left(\mathbf{r}^{\prime}, \tau^{\prime}\right)\right\rangle_{\beta}^{(0)}=\int \frac{(d \mathbf{p})}{(2 \pi)^{3}}[1 \pm n(\mathbf{p})] e^{i \mathbf{p} \cdot\left(\mathbf{r}-\mathbf{r}^{\prime}\right)} e^{-E(\mathbf{p})\left(\tau-\tau^{\prime}\right)}
$$

and

$$
\left\langle\psi^{\dagger}\left(\mathbf{r}^{\prime}, \tau^{\prime}\right) \psi(\mathbf{r}, \tau)\right\rangle_{\beta}^{(0)}=\int \frac{(d \mathbf{p})}{(2 \pi)^{3}} n(\mathbf{p}) e^{i \mathbf{p} \cdot\left(\mathbf{r}-\mathbf{r}^{\prime}\right)} e^{-E(\mathbf{p})\left(\tau-\tau^{\prime}\right)},
$$

where the upper + sign is for Bosons, the lower - sign is for Fermions,

$$
E(\mathbf{p})=\mathbf{p}^{2} / 2 M
$$

is the kinetic energy of the particle with mass $M$, and

$$
n(\mathbf{p})=\frac{1}{e^{-\beta[E(\mathbf{p})-\mu]} \mp 1}
$$

is the momentum distribution for free Bosons or Fermions. Using the results (A25) and (A26), we have

$$
\begin{aligned}
\Pi^{(0)}\left(\mathbf{r}-\mathbf{r}^{\prime}, \tau-\tau^{\prime}\right)=\sum_{s} e_{s}^{2} \int & \frac{(d \mathbf{k})}{(2 \pi)^{3}} e^{i \mathbf{k} \cdot\left(\mathbf{r}-\mathbf{r}^{\prime}\right)} \int \frac{(d \mathbf{p})}{(2 \pi)^{3}} e^{-\left[E_{s}(\mathbf{p}+\mathbf{k})-E_{s}(\mathbf{p})\right]\left(\tau-\tau^{\prime}\right)} \\
& \begin{cases}n_{s}(\mathbf{p})\left[1 \pm n_{s}(\mathbf{p}+\mathbf{k})\right], & \tau>\tau^{\prime} \\
n_{s}(\mathbf{p}+\mathbf{k})\left[1 \pm n_{s}(\mathbf{p})\right], & \tau^{\prime}>\tau\end{cases}
\end{aligned}
$$

The Fourier transform of this expression yields

$$
\Pi^{(0)}\left(k, i \omega_{n}\right)=\sum_{s} e_{s}^{2} \int \frac{(d \mathbf{p})}{(2 \pi)^{3}} \frac{n_{s}(\mathbf{p}+\mathbf{k})-n_{s}(\mathbf{p})}{i \omega_{n}-\left[E_{s}(\mathbf{p}+\mathbf{k})-E_{s}(\mathbf{p})\right]} .
$$

The long-distance behavior of the polarization is controlled by the Debye wave number

$$
\kappa_{D}^{2}=4 \pi \Pi(0,0)
$$

In the one-loop approximation,

\footnotetext{
${ }^{12}$ It is easy to confirm the validity of the results (A25) and (A26): They obey the free field equations of motion, the equal-time commutation or anticommutation relations, and they obey the cyclic boundary condition (A4) generalized to include the number operator and chemical potential in an effective Hamiltonian. These conditions fix the results uniquely.
} 


$$
\begin{aligned}
\kappa_{D}^{2} & \simeq-4 \pi \sum_{s} e_{s}^{2} \int \frac{(d \mathbf{p})}{(2 \pi)^{3}} \frac{\partial n_{s}(\mathbf{p})}{\partial E_{s}(\mathbf{p})} \\
& =4 \pi \sum_{s} e_{s}^{2} \frac{\partial}{\partial \mu_{s}} \int \frac{(d \mathbf{p})}{(2 \pi)^{3}} n_{s}(\mathbf{p})=4 \pi \sum_{s} e_{s}^{2} \frac{\partial}{\partial \mu_{s}}\left\langle n_{s}\right\rangle_{\beta}^{(0)}
\end{aligned}
$$

In the dilute gas limit, the Bose and Fermi distributions can be replaced by the Maxwell distribution

$$
n(\mathbf{p}) \simeq e^{-\beta[E(\mathbf{p})-\mu]}
$$

and in this limit

$$
\kappa_{D}^{2}=4 \pi \beta \sum_{s} e_{s}^{2}\left\langle n_{s}\right\rangle_{\beta}^{(0)}
$$

The Debye wave number is essentially a classical quantity, and in the dilute approximation the zero-frequency classical dielectric function is given by

$$
\epsilon(k, 0)=1+\frac{\kappa_{D}^{2}}{k^{2}}
$$

Placing this in the classical limit (A19) of the charge density correlation function gives

$$
\left\langle\rho(\mathbf{r}) \rho\left(\mathbf{r}^{\prime}\right)\right\rangle_{\beta} \simeq \frac{1}{\beta} \int \frac{(d \mathbf{k})}{(2 \pi)^{3}} \frac{k^{2}}{4 \pi} \frac{\kappa_{D}^{2}}{k^{2}+\kappa_{D}^{2}} e^{i \mathbf{k} \cdot\left(\mathbf{r}-\mathbf{r}^{\prime}\right)} .
$$

The corresponding potential correlation function is thus

$$
\left\langle\phi(\mathbf{r}) \phi\left(\mathbf{r}^{\prime}\right)\right\rangle_{\beta} \simeq \frac{1}{\beta} \int \frac{(d \mathbf{k})}{(2 \pi)^{3}} \frac{4 \pi}{k^{2}} \frac{\kappa_{D}^{2}}{k^{2}+\kappa_{D}^{2}} e^{i \mathbf{k} \cdot\left(\mathbf{r}-\mathbf{r}^{\prime}\right)} .
$$

The partial fraction decomposition

$$
\frac{1}{k^{2}} \frac{\kappa_{D}^{2}}{k^{2}+\kappa_{D}^{2}}=\frac{1}{k^{2}}-\frac{1}{k^{2}+\kappa_{D}^{2}}
$$

expresses the potential correlation function as the difference of the Fourier representations of the Coulomb and Yukawa potentials, and so

$$
\left\langle\phi(\mathbf{r}) \phi\left(\mathbf{r}^{\prime}\right)\right\rangle_{\beta} \simeq \frac{1}{\beta\left|\mathbf{r}-\mathbf{r}^{\prime}\right|}\left[1-\exp \left\{-\kappa_{D}\left|\mathbf{r}-\mathbf{r}^{\prime}\right|\right\}\right]
$$

In the dilute gas limit, $n(\mathbf{p}) \ll 1$ and the Maxwell distribution (A33) applies. Thus, in this limit, and for $\tau>\tau^{\prime}$, the result ( 


$$
\Pi^{(0)}\left(k, \tau-\tau^{\prime}\right) \simeq \sum_{s} e_{s}^{2} e^{\beta \mu_{s}} \int \frac{(d \mathbf{p})}{(2 \pi)^{3}} e^{-\left[E_{s}(\mathbf{p}+\mathbf{k})-E_{s}(\mathbf{p})\right]\left(\tau-\tau^{\prime}\right)} e^{-\beta E_{s}(\mathbf{p})} .
$$

Since the energies that appear in the exponentials are quadratic in the momentum $\mathbf{p}$, the momentum integral is Gaussian which can be evaluated by completing the square. The same considerations apply for the other time order $\tau^{\prime}>\tau$, and one finds that for a dilute gas

$$
\Pi^{(0)}\left(k, \tau-\tau^{\prime}\right) \simeq \sum_{s} e_{s}^{2}\left\langle n_{s}\right\rangle_{\beta}^{(0)} C_{s}\left(k, \tau-\tau^{\prime}\right)
$$

where

$$
C_{s}\left(k, \tau-\tau^{\prime}\right)=\exp \left\{-\frac{k^{2}}{2 M_{s}}\left|\tau-\tau^{\prime}\right|\left[1-\left|\tau-\tau^{\prime}\right| / \beta\right]\right\}
$$

\section{APPENDIX B: DENSITY CALCULATED BY REACTION RATE METHOD}

The method employed in the text to compute the nuclear reaction rate in a plasma may also be used to compute the average density of one of the species of the particles in the plasma. This we shall do here so as to illustrate the method in a simpler context and also to obtain some results that are needed for the work of the text.

Using the techniques of the text, it is easy to see that the average density of a particle with chemical potential $\mu$ may be expressed as

$$
\langle n\rangle_{\beta}=e^{\beta \mu}\left\langle\mathbf{r}^{\prime}=0,-i \beta\left|\langle U(\beta)\rangle_{\beta}\right| \mathbf{r}^{\prime}=0\right\rangle,
$$

where the overall matrix element is a single-particle matrix element of the particle in question and now

$$
U(\beta)=\left(\exp \left\{-\int_{0}^{\beta} d \tau q \phi(\mathbf{r}(\tau), \tau)\right\}\right)_{+},
$$

\footnotetext{
${ }^{13} \mathrm{~A}$ standard treatment of some of the results that we obtain here appears, for example, in Fetter and Walecka (1971), Sec. 30.
} 
with $q$ the charge of the particle and $\mathbf{r}(\tau)$ the particle's coordinate operator which undergoes free motion in the imaginary time $\tau$. The only approximation involved here is the requirement that the particle density $\langle n\rangle_{\beta}$ being computed is dilute. The other components in the plasma may be dense.

To illustrate the method, we shall, however, compute the case in which all the particles in the plasma are dilute so that the exponential in $U(\beta)$ may be expanded with only the first non-vanishing correction retained,

$$
\langle U(\beta)\rangle_{\beta} \simeq 1+\frac{q^{2}}{2} \int_{0}^{\beta} d \tau d \tau^{\prime}\left\langle(\phi(\mathbf{r}(\tau), \tau) \phi(\mathbf{r}(\tau), \tau))_{+}\right\rangle_{\beta} .
$$

Here

$$
\left\langle\left(\phi(\mathbf{r}, \tau) \phi\left(\mathbf{r}^{\prime}, \tau\right)\right)_{+}\right\rangle_{\beta}=\int \frac{(d \mathbf{k})}{(2 \pi)^{3}} e^{i \mathbf{k} \cdot\left(\mathbf{r}-\mathbf{r}^{\prime}\right)} G_{\beta}\left(k, \tau-\tau^{\prime}\right)
$$

with, as is appropriate for the dilute case,

$$
G_{\beta}\left(k, \tau-\tau^{\prime}\right)=\frac{1}{\beta} \sum_{n} e^{-i \omega_{n}\left(\tau-\tau^{\prime}\right)}\left(\frac{4 \pi}{k^{2}}\right)^{2} \frac{\Pi^{(0)}\left(k, i \omega_{n}\right)}{1+\frac{4 \pi}{k^{2}} \Pi^{(0)}\left(k, i \omega_{n}\right)},
$$

where the lowest-order polarization function $\Pi^{(0)}$ has been calculated in Appendix A. The work in the text [c.f. Eq.'s (3.43) and (3.44)] tells us that, with $M$ the mass of the particle in question,

$$
\left\langle\mathbf{r}^{\prime}=0,-i \beta \mid \mathbf{r}^{\prime}=0\right\rangle=\left(\frac{M}{2 \pi \beta}\right)^{3 / 2}
$$

and

$$
\left\langle\mathbf{r}^{\prime}=0,-i \beta\left|\left(e^{i \mathbf{k} \cdot\left[\mathbf{r}(\tau)-\mathbf{r}\left(\tau^{\prime}\right)\right]}\right)_{+}\right| \mathbf{r}^{\prime}=0\right\rangle=\left(\frac{M}{2 \pi \beta}\right)^{3 / 2} C\left(k, \tau-\tau^{\prime}\right)
$$

Here

$$
C\left(k, \tau-\tau^{\prime}\right)=\exp \left\{-\frac{k^{2}}{2 M}\left|\tau-\tau^{\prime}\right|\left[1-\left|\tau-\tau^{\prime}\right| / \beta\right]\right\}
$$

is periodic in the imaginary times $\tau$ and $\tau^{\prime}$ in the interval $0, \beta$ as is the function $G_{\beta}\left(k, \tau-\tau^{\prime}\right)$. Since two such periodic functions may be expressed as Fourier series, the double time integral reduces to a single integral, 


$$
\int_{0}^{\beta} d \tau d \tau^{\prime} f\left(\tau-\tau^{\prime}\right) g\left(\tau-\tau^{\prime}\right)=\beta \int_{0}^{\beta} d \tau f(\tau) g(\tau)
$$

and we may write our result as

$$
\langle n\rangle_{\beta} \simeq e^{\beta \mu}\left(\frac{M}{2 \pi \beta}\right)^{3 / 2}\left\{1+\beta \frac{q^{2}}{2} \int_{0}^{\beta} d \tau \int \frac{(d \mathbf{k})}{(2 \pi)^{3}} C(k, \tau) G_{\beta}(k, \tau)\right\} .
$$

To evaluate the correction which appears here, we set

$$
C(k, \tau)=1+[C(k, \tau)-1]
$$

and note that $[C(k, \tau)-1]$ vanishes as $k^{2}$ for small $k$. Thus the long-ranged contribution of the potential correlation function is reduced in this second term, it is no longer singular at small $k$, and the potential correlation function may be replaced by its zeroth-order value. On the other hand, the replacement of $C(k, \tau)$ by unity just picks out the $n=0$ Fourier mode of the potential correlation function. Therefore, to within an accuracy of order $e^{2}$,

$$
\begin{array}{r}
\int_{0}^{\beta} d \tau \int \frac{(d \mathbf{k})}{(2 \pi)^{3}} C(k, \tau) G_{\beta}(k, \tau)=\int \frac{(d \mathbf{k})}{(2 \pi)^{3}}\left(\frac{4 \pi}{k^{2}}\right) \frac{4 \pi \Pi^{(0)}(k, 0)}{k^{2}+4 \pi \Pi^{(0)}(k, 0)} \\
+\int_{0}^{\beta} d \tau \int \frac{(d \mathbf{k})}{(2 \pi)^{3}}[C(k, \tau)-1]\left(\frac{4 \pi}{k^{2}}\right)^{2} \Pi^{(0)}(k, \tau) .
\end{array}
$$

Recalling that $4 \pi \Pi(0,0)=\kappa_{D}^{2}$ and noting that only potential singular behavior of the integration at small $k$ can give a contribution that is not of order $e^{2}$, we see that to this order we may write

$$
\begin{aligned}
\int \frac{(d \mathbf{k})}{(2 \pi)^{3}}\left(\frac{4 \pi}{k^{2}}\right) \frac{4 \pi \Pi^{(0)}(k, 0)}{k^{2}+4 \pi \Pi^{(0)}(k, 0)} & =\int \frac{(d \mathbf{k})}{(2 \pi)^{3}}\left(\frac{4 \pi}{k^{2}}\right) \frac{\kappa_{D}^{2}}{k^{2}+\kappa_{D}^{2}} \\
& +\int \frac{(d \mathbf{k})}{(2 \pi)^{3}}\left(\frac{4 \pi}{k^{2}}\right)^{2}\left[\Pi^{(0)}(k, 0)-\Pi^{(0)}(0,0)\right]
\end{aligned}
$$

Here

$$
\int \frac{(d \mathbf{k})}{(2 \pi)^{3}}\left(\frac{4 \pi}{k^{2}}\right) \frac{\kappa_{D}^{2}}{k^{2}+\kappa_{D}^{2}}=\kappa_{D}
$$

while

$$
\begin{aligned}
\int \frac{(d \mathbf{k})}{(2 \pi)^{3}}\left(\frac{4 \pi}{k^{2}}\right)^{2}\left[\Pi^{(0)}(k, 0)-\Pi^{(0)}(0,0)\right] & =-8 \int_{0}^{\infty} d k\left[\Pi^{(0)}(k, 0)-\Pi^{(0)}(0,0)\right] \frac{d}{d k} \frac{1}{k} \\
& =8 \int_{0}^{\beta} d \tau \int_{0}^{\infty} d k \frac{1}{k} \frac{d}{d k} \Pi^{(0)}(k, \tau)
\end{aligned}
$$


where we have used polar coordinates, integrated by parts, and re-expressed the zero frequency component of the polarization function as a integral over imaginary time. The results that we have just obtained may be restated in the form used in Section 3.2 of the text,

$$
\int_{0}^{\beta} d \tau \int \frac{(d \mathbf{k})}{(2 \pi)^{3}} G_{\beta}(k, \tau)=\kappa_{D}+8 \int_{0}^{\beta} d \tau \int_{0}^{\infty} d k \frac{1}{k} \frac{d}{d k} \Pi^{(0)}(k, \tau)
$$

which we reiterate is accurate to terms including order $e^{2}$.

For the second set of terms in Eq. (B12), we again integrate by parts as was done in Eq. (B15) to obtain

$$
\begin{aligned}
& \int_{0}^{\beta} d \tau \int \frac{(d \mathbf{k})}{(2 \pi)^{3}}[C(k, \tau)-1]\left(\frac{4 \pi}{k^{2}}\right)^{2} \Pi^{(0)}(k, \tau)= \\
& \quad 8 \int_{0}^{\beta} d \tau \int_{0}^{\infty} d k \frac{1}{k}\left\{\frac{d}{d k}\left[C(k, \tau) \Pi^{(0)}(k, \tau)\right]-\frac{d}{d k} \Pi^{(0)}(k, \tau)\right\}
\end{aligned}
$$

The second term in the integrand on the right-hand side of this equation just cancels the contribution in Eq. (B15). For the first term in the integrand, we recall the results (A41) and (A42) from Appendix A which give the dilute gas form

$$
\Pi^{(0)}(k, \tau)=\sum_{s} e_{s}^{2}\left\langle n_{s}\right\rangle_{\beta}^{(0)} \exp \left\{-\frac{k^{2}}{2 M_{s}} \tau[1-\tau / \beta]\right\} .
$$

The exponential here is of the same form as that in the definition (B8) of $C(k, \tau)$, and the two combine to form a single such exponential which involves the reduced mass

$$
\frac{1}{\bar{M}_{s}}=\frac{1}{m}+\frac{1}{M_{s}}
$$

Thus,

$$
\begin{aligned}
8 \int_{0}^{\beta} d \tau \int_{0}^{\infty} & d k \frac{1}{k} \frac{d}{d k}\left[C(k, \tau) \Pi^{(0)}(k, \tau)\right] \\
& =-8 \sum_{s} e_{s}^{2}\left\langle n_{s}\right\rangle_{\beta}^{(0)} \int_{0}^{\beta} d \tau \int_{0}^{\infty} d k\left\{\frac{1}{\bar{M}_{s}} \tau[1+\tau / \beta]\right\} \exp \left\{-\frac{\mathbf{k}^{2}}{2 \bar{M}_{s}} \tau[1-\tau / \beta]\right\} \\
& =-4 \sum_{s} e_{s}^{2}\left\langle n_{s}\right\rangle_{\beta}^{(0)} \int_{0}^{\beta} d \tau \sqrt{\frac{2 \pi}{\bar{M}_{s}} \tau[1+\tau / \beta]} \\
& =-\frac{1}{8} \beta \sum_{s} 4 \pi e_{s}^{2}\left\langle n_{s}\right\rangle_{\beta}^{(0)} \sqrt{\frac{2 \pi \beta}{\bar{M}_{s}}}
\end{aligned}
$$

We write 


$$
\kappa_{D, s}^{2}=4 \pi e_{s}^{2}\left\langle n_{s}\right\rangle_{\beta},
$$

which is the contribution of the plasma species $s$ to the squared Debye wave number, and

$$
\bar{\lambda}_{s}=\sqrt{\frac{2 \pi \beta}{\bar{M}_{s}}}
$$

which is the thermal wave length of a particle with the reduced mass $\bar{M}_{s}$. With these definitions, our evaluation reads

$$
8 \int_{0}^{\beta} d \tau \int_{0}^{\infty} d k \frac{1}{k} \frac{d}{d k}\left[C(k, \tau) \Pi^{(0)}(k, \tau)\right]=-\frac{1}{8} \beta \sum_{s} \kappa_{D, s}^{2} \bar{\lambda}_{s}
$$

Collecting the results finally yields

$$
\langle n\rangle_{\beta}=e^{\beta \mu}\left(\frac{M}{2 \pi \beta}\right)^{3 / 2}\left\{1+\frac{1}{2} \beta q^{2} \kappa_{D}-\frac{\beta q^{2}}{16} \sum_{s} \kappa_{D, s}^{2} \bar{\lambda}_{s}\right\} .
$$

This result is accurate including terms of order $e^{4}$. The first correction is the classical correction, and it is of order $e^{3}$. The second correction, of order $e^{4}$, is of a quantum nature. It involves the quantum wave length $\bar{\lambda}_{s}$ which, with ordinary units, is proportional to $\hbar$.

The light electron has a thermal wave length $\lambda_{e}$ that is much larger than the thermal wave lengths $\lambda_{s}$ of the nuclear particles in the plasma. Thus, the electron provides the largest quantum correction in the result (B24). The large thermal wave length of the electron, however, also implies that the chemical potential factor $\exp \left\{\beta \mu_{e}\right\}$ for the electron is much larger than those of the other plasma particles since electrical neutrality requires that half the particles in the plasma be electrons but their number density is proportional to $\lambda_{e}^{-3}$. Thus it may well not be a good approximation to treat the electron distribution as a dilute, Maxwell-Boltzmann distribution. On the other hand, the small electron mass implies that wave numbers $k$ that are very small in comparison to $\sqrt{M_{s} / \beta}$ dominate in the electron contribution to Eq. (B20), and so for their contribution we may write

$$
C(k, \tau) \simeq 1
$$

up to a correction of relative order $m_{e} / M_{s}$. In this case, only the zero frequency mode of the electron polarization function appears, and the contribution of this function can easily 
be evaluated since the electron Fermi-Dirac distribution may be written as a superposition of Maxwell-Boltzmann distributions,

$$
\frac{1}{e^{\beta\left[E_{e}(p)-\mu_{e}\right]}+1}=\sum_{n=1}^{\infty}(-1)^{n+1} e^{-n \beta\left[E_{e}(p)-\mu_{e}\right]} .
$$

Thus the electron contribution to Eq. (B20) may be expressed as an infinite sum of the Maxwell distributions already used in the previous evaluation, and we have

$$
\begin{aligned}
8 \int_{0}^{\beta} d \tau \int_{0}^{\infty} d k \frac{1}{k} \frac{d}{d k} \Pi_{e}^{(0)}(k, \tau) & =8 \sum_{n=1}^{\infty}(-1)^{n+1} \int_{0}^{n \beta} d \tau \int_{0}^{\infty} d k \frac{1}{k} \frac{d}{d k} \Pi^{(\mathrm{M}-\mathrm{B})}(k, \tau ; n \beta) \\
& =-\frac{\pi e^{2}}{2} \sum_{n=1}^{\infty}(-1)^{n+1}\left\langle n_{e}\right\rangle_{n \beta}^{(\mathrm{M}-\mathrm{B})} n \beta \sqrt{\frac{2 \pi n \beta}{m_{e}}} \\
& =-\frac{1}{4} e^{2} m_{e} \sum_{n=1}^{\infty}(-1)^{n+1} 2 e^{n \beta \mu_{e}} \\
& =-\frac{1}{4} e^{2} m_{e} \frac{2}{e^{-\beta \mu_{e}}+1},
\end{aligned}
$$

where the extra factor of 2 accounts for the two spin polarizations of the electron. Using this more accurate treatment for the electrons and noting that

$$
a_{0}=\frac{1}{e^{2} m_{e}}
$$

is the electron Bohr radius, we find that

$$
\langle n\rangle_{\beta}=e^{\beta \mu}\left(\frac{M}{2 \pi \beta}\right)^{3 / 2}\left\{1+\frac{1}{2} \beta q^{2} \kappa_{D}-\frac{\beta q^{2}}{16} \sum_{s \neq e} \kappa_{D, s}^{2} \bar{\lambda}_{s}-\frac{1}{4} \beta \frac{q^{2}}{a_{0}} \frac{1}{e^{-\beta \mu_{e}}+1}\right\} .
$$

It should be recalled that we consider different spin states as separate species; the total number density of a spin $1 / 2$ species is given by twice this result.

\section{APPENDIX C: CLASSICAL PLASMA}

As discussed in the text, the correction due to the ionic component of the plasma cannot be evaluated using a classical treatment for the plasma unless its thermal wavelength is much less than the turning radius of the reacting particles. In the text, we extracted this limit from our general result for a weakly coupled plasma. Here we shall show how this result is 
obtained when one starts with a formulation that is often employed in the literature. This formulation neglects the motion of the center of mass and takes the reaction rate to be proportional to the single-particle, relative motion quantum-mechanical expectation value

$$
\begin{array}{r}
\left\langle\left\langle\check{\mathbf{r}}^{\prime}=0\left|\exp \left\{-\beta\left[H_{r}+e_{1} \phi\left(\check{\mathbf{r}} M_{2} / M\right)+e_{2} \phi\left(\check{\mathbf{r}} M_{1} / M\right)\right]\right\}\right| \check{\mathbf{r}}^{\prime}=0\right\rangle\right\rangle_{\beta}^{\mathrm{cl}} \propto \\
\left(\mid\left\langle\left(\exp \left\{-\int_{0}^{\beta} d \tau\left[e_{1} \phi\left(\check{\mathbf{r}}(\tau) M_{2} / M\right)+e_{2} \phi\left(\check{\mathbf{r}}(\tau) M_{1} / M\right)\right]\right)_{+}\right\rangle_{\beta}^{\mathrm{cl}}\right|\right),
\end{array}
$$

where we have passed to the interaction picture on the right-hand side of the equation $[c . f$. Eq. (2.46)] and used the normalized expectation value defined in Eq. (3.46). Here classical statistical mechanics is used to evaluate the thermal expectation value. This formula is often used in the literature to obtain an effective potential for the quantum-mechanical relative motion, and then the tunneling problem which describes the quantum expectation value is solved for this effective potential. We shall content ourselves by showing that this procedure gives our previous limit for the first plasma correction when the plasma is dilute. Using the result (A39) for the classical thermal expectation value of the potential correlation function and expanding out to the first correction, we get terms of the form

$$
\begin{aligned}
& 1+\frac{1}{2} \int_{0}^{\beta} d \tau d \tau^{\prime}\left\{e_{1}^{2}\left\langle\phi\left(\check{\mathbf{r}}(\tau) M_{2} / M\right) \phi\left(\check{\mathbf{r}}\left(\tau^{\prime}\right) M_{2} / M\right)\right\rangle_{\beta}^{\mathrm{cl}}+\cdots\right\}=1+ \\
& \frac{1}{2} \int_{0}^{\beta} d \tau d \tau^{\prime}\left\{e_{1}^{2} \frac{M_{2} / M}{\beta\left|\check{\mathbf{r}}(\tau)-\check{\mathbf{r}}\left(\tau^{\prime}\right)\right|}\left[1-\exp \left\{-\kappa_{D}\left|\check{\mathbf{r}}(\tau)-\check{\mathbf{r}}\left(\tau^{\prime}\right)\right| M_{2} / M\right\}\right]+\cdots\right\} .
\end{aligned}
$$

Expanding in powers of the Debye wave number, which is equivalent to expanding in the small parameter $\kappa_{D} r_{\max }$, and adding up all the terms produces

$$
\begin{array}{rl}
1+\frac{1}{2}\left(e_{1}+e_{2}\right)^{2} \beta \kappa_{D}-\frac{\kappa_{D}^{2}}{4 \beta} \int_{0}^{\beta} d & d \tau^{\prime}\left\{\left(e_{1}^{2} \frac{M_{2}}{M}+e_{2}^{2} \frac{M_{1}}{M}\right)\left|\check{\mathbf{r}}(\tau)-\check{\mathbf{r}}\left(\tau^{\prime}\right)\right|\right. \\
& \left.+2 e_{1} e_{2}\left|\frac{M_{2}}{M} \check{\mathbf{r}}(\tau)+\frac{M_{1}}{M} \check{\mathbf{r}}\left(\tau^{\prime}\right)\right|\right\}
\end{array}
$$

This is precisely the classical plasma limit that was discussed in the text following Eq. (3.71). 


\section{APPENDIX D: REAL TIME TROUBLES}

The work in the text made use of thermodynamic, "imaginary time" methods. Here we shall compare and contrast this method with the formulation which employs purely real time methods. The real time method might appear have the advantage of displaying the dynamics of the reaction process in terms of simple physical pictures, such as that proposed by Carrero, Schäfer, and Koonin (1988). We shall show explicitly, however, that this is an

illusion. Terms in the real time formulation that apparently have a straight forward physical interpretation may, in fact, be completely cancelled out by other terms. In particular, we shall show that the correction found by Carrero et al. has such a cancelation and hence does not exist.

To relate the two formulations, we shall pass to an interaction picture. This is done by partitioning the complete Hamiltonian $H$ of the total system into a part $H_{0}$ that describes the dynamics of the background plasma and the reacting particles, but with no interactions between the reacting particles and the plasma, and the remaining part $H_{1}$ that the describes the interactions of the reacting particles with the background plasma,

$$
H=H_{0}+H_{1}
$$

The interaction picture is obtained by writing

$$
e^{-i H\left(t_{1}-t_{2}\right)}=e^{-i H_{0} t_{1}} U_{+}\left(t_{1}, t_{2}\right) e^{i H_{0} t_{2}},
$$

where

$$
U_{+}\left(t_{1}, t_{2}\right)=\left(\exp \left\{-i \int_{t_{1}}^{t_{2}} d t H_{1}(t)\right\}\right)_{+}
$$

involves the time-ordered exponential with

$$
H_{1}(t)=e^{i H_{0} t} H_{1} e^{-i H_{0} t}
$$

The interaction picture time evolution operator is unitary, 


$$
U_{+}\left(t_{1}, t_{2}\right)^{\dagger}=U_{+}\left(t_{1}, t_{2}\right)^{-1},
$$

and obeys the group property,

$$
U_{+}\left(t_{1}, t_{3}\right) U_{+}\left(t_{3}, t_{2}\right)=U_{+}\left(t_{1}, t_{2}\right) .
$$

The general expression for the reaction rate was derived in the text and presented in Eq. (2.10), which we repeat here for convenience:

$$
\begin{aligned}
\Gamma & =\int_{-\infty}^{+\infty} d t \int(d \mathbf{r})\left\langle\mathcal{K}^{\dagger}(\mathbf{r}, t) \mathcal{K}(0)\right\rangle_{\beta} \\
& =g^{2} \int_{-\infty}^{+\infty} d t \int(d \mathbf{r}) e^{i Q t}\left\langle\psi_{1}^{\dagger}(\mathbf{r}, t) \psi_{2}^{\dagger}(\mathbf{r}, t) \psi_{3}(\mathbf{r}, t) \psi_{4}(\mathbf{r}, t) \psi_{4}^{\dagger}(0) \psi_{3}^{\dagger}(0) \psi_{2}(0) \psi_{1}(0)\right\rangle_{\beta} .
\end{aligned}
$$

Using the interaction picture decomposition (D2) for the statistical operator $\exp \{-\beta H\}$ which is the weight in the thermal expectation value $\langle\cdots\rangle_{\beta}$ and also using this decomposition for the time dependence of $\mathcal{K}^{\dagger}(\mathbf{r}, t)$ produces

$$
\Gamma=\int_{-\infty}^{+\infty} d t \int(d \mathbf{r})\left\langle U_{+}(-i \beta, 0) U_{+}^{-1}(t, 0) \mathcal{K}^{\dagger}(\mathbf{r}, t) U_{+}(t, 0) \mathcal{K}(0)\right\rangle_{\beta}^{I} .
$$

Here the superscript $I$ indicates that the time dependence is now governed by $H_{0}$ and also that the statistical ensemble is now described (except for the full normalizing partition function in the denominator) by $\exp \left\{-\beta H_{0}\right\}$. This is the "imaginary time" formulation that was essentially employed in the text.

In the real time formulation, one computes the thermal average of the square of corrected matrix elements and thus arrives at

$$
\begin{aligned}
\Gamma=\int_{-\infty}^{+\infty} d t \int(d \mathbf{r})\left\langle\left[U_{+}^{\dagger}(t,-\infty) \mathcal{K}^{\dagger}(\mathbf{r}, t) U_{+}^{\dagger}(+\infty, t)\right]\right. \\
{\left.\left[U_{+}(+\infty, 0) \mathcal{K}(0) U_{+}(0,-\infty)\right]\right\rangle_{\beta}^{I} . }
\end{aligned}
$$

To prove that this is indeed the same as the previous result (D8), we make use of the group property (D6) and unitarity (D5) to write Eq. (D9) as

$$
\Gamma=\int_{-\infty}^{+\infty} d t \int(d \mathbf{r})\left\langle U_{+}^{\dagger}(0,-\infty) U_{+}(t, 0)^{-1} \mathcal{K}^{\dagger}(\mathbf{r}, t) U_{+}^{\dagger}(t, 0) \mathcal{K}(0) U_{+}(0,-\infty)\right\rangle_{\beta}^{I} .
$$


Using the cyclic symmetry of the trace which defines the thermal average, we encounter

$$
U_{+}(0,-\infty) e^{-\beta H_{0}} U_{+}^{-1}(0,-\infty)=e^{-\beta H_{0}} U_{+}(-i \beta,-\infty-i \beta) U_{+}^{-1}(0,-\infty) .
$$

It is implicit in the interaction picture representation that the interaction is adiabatically

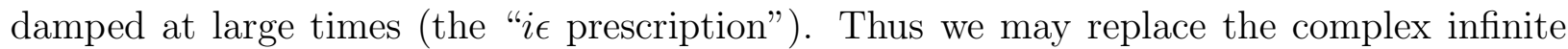
time limit $-\infty-i \beta$ by the real limit $-\infty$ and use the group property (D6) to conclude that

$$
U_{+}(0,-\infty) e^{-\beta H_{0}} U_{+}^{-1}(0,-\infty)=e^{-\beta H_{0}} U_{+}(-i \beta, 0) .
$$

Using these results in Eq. (D10) reduces it to the imaginary time form (D8) and proves its equivalence with the real time form (D9).

To illustrate the differences in the real and imaginary time formulations, we shall examine a simple model in some detail using the methods of both forms. In this model, all the interactions of the final, produced particles are neglected (which may be done if the energy release $Q$ is large). Thus, in this model,

$$
H_{1}=\int(d \mathbf{r})\left[\rho_{1} \phi+\rho_{2} \phi\right]
$$

where $\rho_{1}$ and $\rho_{2}$ are the charge density operators of the initial particles labeled by 1 and 2 . To simplify the exposition, we shall also assume that one of the initial particles, say 2 , is very massive. Thus, except for its ordering position, the charge density operator $\rho_{2}$ is independent of time. To further simplify the model, we shall neglect the Coulomb interactions between the initial particles 1,2 and compute only the correction involving a single interaction between each initial particle and the background plasma, the term proportional to $e_{1} e_{2}$ which we denote by $\Gamma_{12}$. Finally, in our model we shall take the limit in which the background plasma is treated classically. In this classical limit, the wave number vector $\mathbf{k}$ of a plasma correlation function is generally neglected relative to an initial particle momentum $\mathbf{p}$ since these enter in the combination $\mathbf{p}+\hbar \mathbf{k}$. As we shall see, this neglect of the plasma wave number may be done and gives the classical limit except when energy corrections are involved which lead to frequency shifts depending upon $\Delta E / \hbar$. 
We first compute the $e_{1} e_{2}$ term in the imaginary time formulation (D8). As discussed in the text, the corrections associated with the time evolution operator $U_{+}(t, 0)$ are negligible. We neglect these here and compute only those associated with the statistical factor $U_{+}(-i \beta, 0)$,

$$
\begin{aligned}
\Gamma_{12}=\int_{-\infty}^{+\infty} d t & \int(d \mathbf{r}) \int_{0}^{\beta} d \tau_{1} d \tau_{2} \int\left(d \mathbf{r}_{1}\right)\left(d \mathbf{r}_{2}\right)\left\langle\left(\phi\left(\mathbf{r}_{1}, \tau_{1}\right) \phi\left(\mathbf{r}_{2}, \tau_{2}\right)\right)_{+}\right\rangle_{\beta} \\
& \left\langle\left(\rho_{1}\left(\mathbf{r}_{1}, \tau_{1}\right) \rho_{2}\left(\mathbf{r}_{2}, \tau_{2}\right)\right)_{+} \mathcal{K}^{\dagger}(\mathbf{r}, t) \mathcal{K}(0)\right\rangle_{\beta}^{I} .
\end{aligned}
$$

Since the interaction between particles 1 and 2 is neglected, the charge density operators $\rho_{1}$ and $\rho_{2}$ commute. Hence, since $\rho_{2}$ is time independent, the time-ordered product of the two charge density operators is independent of $\tau_{2}$. In view of the discussion of Appendix A, the correction (D14) thus involves

$$
\begin{aligned}
\int_{0}^{\beta} d \tau_{2}\left\langle\left(\phi\left(\mathbf{r}_{1}, \tau_{1}\right) \phi\left(\mathbf{r}_{2}, \tau_{2}\right)\right)_{+}\right\rangle_{\beta} & =\int \frac{(d \mathbf{k})}{(2 \pi)^{3}} \frac{4 \pi}{k^{2}}\left[1-\frac{1}{\epsilon(k, 0)}\right] e^{i \mathbf{k} \cdot\left(\mathbf{r}_{1}-\mathbf{r}_{2}\right)} \\
& =\beta\left\langle\phi\left(\mathbf{r}_{1}\right) \phi\left(\mathbf{r}_{2}\right)\right\rangle_{\beta}^{\mathrm{cl}}
\end{aligned}
$$

where in the second line we have written the classical limit. We stress that, without any further approximation, this correction entails the dielectric permittivity of the plasma $\epsilon(k, \omega)$ at zero frequency, $\omega=0$. That is, the correction depends only upon the static properties of the plasma. Now, in the classical limit, the plasma wave number $\mathbf{k}$ is neglected, and the correction entails only the total charge operators of the initial particles,

$$
\int\left(d \mathbf{r}_{1}\right) \rho_{1}\left(\mathbf{r}_{1}, \tau\right)=Q_{1}, \quad \int\left(d \mathbf{r}_{2}\right) \rho_{2}\left(\mathbf{r}_{2}, \tau\right)=Q_{2},
$$

which are time independent. In the thermal expectation value in Eq. (D14), these operators just measure the charge (with the Boltzmann statistics for the dilute initial particles which we always take), and so we may make the replacements $Q_{1} \rightarrow e_{1}$ and $Q_{2} \rightarrow e_{2}$ to obtain

$$
\Gamma_{12}=e_{1} e_{2} \beta^{2}\langle\phi(\mathbf{0}) \phi(\mathbf{0})\rangle_{\beta}^{\mathrm{cl}} \Gamma_{0},
$$

where $\Gamma_{0}$ is the free gas reaction rate. The classical correlation function for a dilute plasma gives, according to Eq. (A39), 


$$
\beta\langle\phi(\mathbf{0}) \phi(\mathbf{0})\rangle_{\beta}^{\mathrm{cl}}=\kappa_{D},
$$

and thus we arrive at the basic Salpeter correction,

$$
\Gamma_{12}=e_{1} e_{2} \beta \kappa_{D} \Gamma_{0}
$$

Let us note for future reference that, as discussed in the Introduction,

$$
U_{12}^{\text {static pol }}=-e_{1} e_{2} \kappa_{D}
$$

is just the static polarization energy of the plasma induced when the two initial particles are on top of one another. Thus the Salpeter result may be written as

$$
\Gamma_{12}=-\beta U_{12}^{\text {static pol }} \Gamma_{0}
$$

We turn now to compute the $e_{1} e_{2}$ term from the real time form (D9), which gives, on reading from right to left,

$$
\begin{aligned}
\Gamma_{12}= & \int_{-\infty}^{+\infty} d t \int(d \mathbf{r}) \int_{-\infty}^{+\infty} d t_{1} d t_{2} \int\left(d \mathbf{r}_{1}\right)\left(d \mathbf{r}_{2}\right) \\
& \left\{-\left\langle\left(\phi\left(\mathbf{r}_{1}, t_{1}\right) \phi\left(\mathbf{r}_{2}, t_{2}\right)\right)_{+}\right\rangle_{\beta}\left\langle\mathcal{K}^{\dagger}(\mathbf{r}, t)\left(\rho_{1}\left(\mathbf{r}_{1}, t_{1}\right) \rho_{2}\left(\mathbf{r}_{2}, t_{2}\right) \mathcal{K}(0)\right)_{+}\right\rangle_{\beta}^{I}\right. \\
& +\left\langle\phi\left(\mathbf{r}_{2}, t_{2}\right) \phi\left(\mathbf{r}_{1}, t_{1}\right)\right\rangle_{\beta}\left\langle\left(\rho_{2}\left(\mathbf{r}_{2}, t_{2}\right) \mathcal{K}^{\dagger}(\mathbf{r}, t)\right)_{-}\left(\rho_{1}\left(\mathbf{r}_{1}, t_{1}\right) \mathcal{K}(0)\right)_{+}\right\rangle_{\beta}^{I} \\
& +\left\langle\phi\left(\mathbf{r}_{1}, t_{1}\right) \phi\left(\mathbf{r}_{2}, t_{2}\right)\right\rangle_{\beta}\left\langle\left(\rho_{1}\left(\mathbf{r}_{1}, t_{1}\right) \mathcal{K}^{\dagger}(\mathbf{r}, t)\right)_{-}\left(\rho_{2}\left(\mathbf{r}_{2}, t_{2}\right) \mathcal{K}(0)\right)_{+}\right\rangle_{\beta}^{I} \\
& \left.-\left\langle\left(\phi\left(\mathbf{r}_{1}, t_{1}\right) \phi\left(\mathbf{r}_{2}, t_{2}\right)\right)_{-}\right\rangle_{\beta}\left\langle\left(\rho_{1}\left(\mathbf{r}_{1}, t_{1}\right) \rho_{2}\left(\mathbf{r}_{2}, t_{2}\right) \mathcal{K}^{\dagger}(\mathbf{r}, t)\right)_{-} \mathcal{K}(0)\right\rangle_{\beta}^{I}\right\},
\end{aligned}
$$

where the notation $(\cdots)_{-}$denotes the anti-time-ordered product. To simplify the work, we immediately take the classical limit in which the momentum transfer $\hbar \mathbf{k}$ imparted by the plasma to the heavy particle charge density $\rho_{2}$ vanishes. In this limit, the integration over $\mathbf{r}_{2}$ in Eq. (D22) gives the total charge $Q_{2}\left(t_{2}\right)$ which is time independent save for its ordering. With the Boltzmann statistics that we use for the reacting particles 1,2, $Q_{2}$ must appear to the left of the creation operator $\psi_{2}^{\dagger}$ and to the right of the destruction operator $\psi_{2}$, and in these cases it is simply replaced by $e_{2}$. This restriction of the ordering of $Q_{2}\left(t_{2}\right)$ limits the 
time interval over which $t_{2}$ runs to $-\infty<t_{2}<0$ or $-\infty<t_{2}<t$. The interval from 0 to $t$ described by the time evolution operator $U_{+}(t, 0)$ will be neglected here just has it has been previously. With the Boltzmann statistics, a similar time restriction obtains for $\rho\left(\mathbf{r}_{1}, t_{1}\right)$, $-\infty<t_{1}<0$. Thus, the real time result (D22) reduces to

$$
\begin{aligned}
\Gamma_{12}=\int_{-\infty}^{+\infty} d t & \int(d \mathbf{r}) \int_{-\infty}^{0} d t_{1} \int_{-\infty}^{0} d t_{2} \int\left(d \mathbf{r}_{1}\right) \\
\{ & -\left\langle\left(\phi\left(\mathbf{r}_{1}, t_{1}\right) \phi\left(\mathbf{0}, t_{2}\right)\right)_{+}\right\rangle_{\beta} e_{2}\left\langle\mathcal{K}^{\dagger}(\mathbf{r}, t) \mathcal{K}(0) \rho_{1}\left(\mathbf{r}_{1}, t_{1}\right)\right\rangle_{\beta}^{I} \\
& +\left\langle\phi\left(\mathbf{0}, t_{2}\right) \phi\left(\mathbf{r}_{1}, t_{1}\right)\right\rangle_{\beta} e_{2}\left\langle\mathcal{K}^{\dagger}(\mathbf{r}, t) \mathcal{K}(0) \rho_{1}\left(\mathbf{r}_{1}, t_{1}\right)\right\rangle_{\beta}^{I} \\
& +\left\langle\phi\left(\mathbf{r}_{1}, t_{1}\right) \phi\left(\mathbf{0}, t_{2}\right)\right\rangle_{\beta} e_{2}\left\langle\rho_{1}\left(\mathbf{r}_{1}, t_{1}\right) \mathcal{K}^{\dagger}(\mathbf{r}, t) \mathcal{K}(0)\right\rangle_{\beta}^{I} \\
& \left.-\left\langle\left(\phi\left(\mathbf{r}_{1}, t_{1}\right) \phi\left(\mathbf{0}, t_{2}\right)\right)_{-}\right\rangle_{\beta} e_{2}\left\langle\rho_{1}\left(\mathbf{r}_{1}, t_{1}\right) \mathcal{K}^{\dagger}(\mathbf{r}, t) \mathcal{K}(0)\right\rangle_{\beta}^{I}\right\} .
\end{aligned}
$$

The terms that appear in the successive lines on the right-hand side of this equation appear to have a clear physical description. The first line corresponds to the correction that results when the initial particles 1 and 2 have a Coulomb exchange which is modified by the plasma in a dynamical (frequency or time dependent) fashion before the fusion reaction takes place. The last line give the same correction for the complex conjugate amplitude that enters into the squared matrix element. The second and third lines do not describe such second-order interactions which take place between the initial particles before the fusion occurs. They instead describe the square of first-order amplitudes. They correspond to processes in which, if a complete set of final plasma states is introduced between $\mathcal{K}^{\dagger}$ and $\mathcal{K}$, the plasma is left in excited states. In one of the amplitudes, this excitation is caused by particle 1 , in the other, by particle 2. The trouble with the real-time formulation is that there is extensive cancelation between these terms that might appear to have a distinct physical meaning.

To make a first combination of terms, we write

$$
\left\langle\left(\phi(\mathbf{r}, t) \phi\left(\mathbf{r}^{\prime}, t^{\prime}\right)\right)_{ \pm}\right\rangle_{\beta}= \pm \frac{1}{2} \epsilon\left(t-t^{\prime}\right)\left\langle\left[\phi(\mathbf{r}, t), \phi\left(\mathbf{r}^{\prime}, t^{\prime}\right)\right]\right\rangle_{\beta}+\frac{1}{2}\left\langle\left\{\phi(\mathbf{r}, t), \phi\left(\mathbf{r}^{\prime}, t^{\prime}\right)\right\}\right\rangle_{\beta},
$$

where the curly brackets denote the anticommutator, and

$$
\left\langle\phi(\mathbf{r}, t) \phi\left(\mathbf{r}^{\prime}, t^{\prime}\right)\right\rangle_{\beta}=\frac{1}{2}\left\langle\left[\phi(\mathbf{r}, t), \phi\left(\mathbf{r}^{\prime}, t^{\prime}\right)\right]\right\rangle_{\beta}+\frac{1}{2}\left\langle\left\{\phi(\mathbf{r}, t), \phi\left(\mathbf{r}^{\prime}, t^{\prime}\right)\right\}\right\rangle_{\beta} .
$$


The terms involving the anticommutator pieces in Eq. (D23) all cancel. As discussed in Appendix A, the commutator has the thermal expectation value

$$
\left\langle\left[\phi(\mathbf{r}, t), \phi\left(\mathbf{r}^{\prime}, t^{\prime}\right)\right]\right\rangle_{\beta}=\int \frac{(d \mathbf{k})}{(2 \pi)^{3}} \int \frac{d \omega^{\prime}}{2 \pi}\left(\frac{4 \pi}{k^{2}}\right)^{2} c\left(k, \omega^{\prime}\right) e^{i \mathbf{k} \cdot\left(\mathbf{r}-\mathbf{r}^{\prime}\right)-i \omega^{\prime}\left(t-t^{\prime}\right)} .
$$

Appendix A also shows that $c\left(k, \omega^{\prime}\right)$ appears as the spectral weight which defines the dielectric function $\epsilon(k, \omega)$ by the dispersion relation

$$
\frac{1}{\epsilon(k, \omega)}-1=\frac{4 \pi}{k^{2}} \int \frac{d \omega^{\prime}}{2 \pi} \frac{c\left(k, \omega^{\prime}\right)}{\omega-\omega^{\prime}-i \epsilon},
$$

a result that we shall soon make use of.

Making use of Eq's. (A25) and (A26) in the Boltzmann statistics limit in which $n(\mathbf{p})$ is neglected relative to 1 , and neglecting the plasma wave number $\mathbf{k}$ in spatial momentum terms, but not in the energies as is appropriate to the limit in which the plasma in treated classically, the first two lines of the right-hand side of Eq.(D23) involve

$$
\int\left(d \mathbf{r}_{1}\right) e^{i \mathbf{k} \cdot \mathbf{r}_{1}}\left\langle\psi_{1}^{\dagger}(\mathbf{r}, t) \psi_{1}(0) \rho_{1}\left(\mathbf{r}_{1}, t_{1}\right)\right\rangle_{\beta}=e_{1} \int \frac{(d \mathbf{p})}{(2 \pi)^{3}} n_{1}(\mathbf{p}) e^{-i \mathbf{p} \cdot \mathbf{r}+i E_{1}(\mathbf{p}) t} e^{i \Delta E t_{1}}
$$

and the second two lines entail

$$
\int\left(d \mathbf{r}_{1}\right) e^{i \mathbf{k} \cdot \mathbf{r}_{1}}\left\langle\rho_{1}\left(\mathbf{r}_{1}, t_{1}\right) \psi_{1}^{\dagger}(\mathbf{r}, t) \psi_{1}(0)\right\rangle_{\beta}=e_{1} \int \frac{(d \mathbf{p})}{(2 \pi)^{3}} n_{1}(\mathbf{p}+\mathbf{k}) e^{-i \mathbf{p} \cdot \mathbf{r}+i E_{1}(\mathbf{p}) t} e^{i \Delta E t_{1}}
$$

in which

$$
\begin{aligned}
\Delta E & =E_{1}(\mathbf{p}+\mathbf{k})-E_{1}(\mathbf{p})=\frac{2 \mathbf{p} \cdot \mathbf{k}+\mathbf{k}^{2}}{2 m_{1}} \\
& \simeq \frac{\mathbf{p} \cdot \mathbf{k}}{m_{1}}=\mathbf{v}_{1} \cdot \mathbf{k}
\end{aligned}
$$

where the approximate equality applies to our classical limit. In view of these results and the

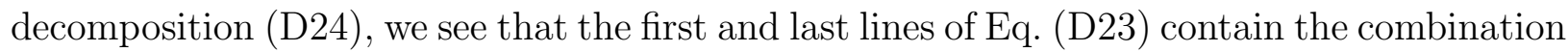

$$
-n_{1}(\mathbf{p})+n_{1}(\mathbf{p}+\mathbf{k})=-e^{-\beta E(\mathbf{p})}+e^{-\beta E(\mathbf{p}+\mathbf{k})} \simeq-\beta \Delta E n_{1}(\mathbf{p})
$$

where, as always, we use Boltzmann statistics for the initial reacting particles, and the last approximation is appropriate for our classical plasma limit. The first and last lines on the right-hand side of Eq. (D23) are evaluated with the time integrals 


$$
\int_{-\infty}^{0} d t_{1} \int_{-\infty}^{0} d t_{2} \epsilon\left(t_{1}-t_{2}\right) e^{-i \omega^{\prime}\left(t_{1}-t_{2}\right)} e^{i \Delta E t_{1}}=\frac{1}{\Delta E}\left[\frac{1}{\Delta E-\omega^{\prime}-i \epsilon}+\frac{1}{-\omega^{\prime}}\right] .
$$

The integration of the denominators which appear here over $\omega^{\prime}$ with the weight $c\left(k, \omega^{\prime}\right)$ is just the dispersion relation (D27), and we see that the first and last lines of Eq. (D23) produce $\beta$ times the factor

$$
-U_{12}^{\text {dyn pol }}\left(\mathbf{v}_{1}\right)=-\frac{1}{2} e_{1} e_{2} \int \frac{(d \mathbf{k})}{(2 \pi)^{3}} \frac{4 \pi}{k^{2}}\left\{\left[\frac{1}{\epsilon\left(k, \mathbf{v}_{1} \cdot \mathbf{k}\right)}-1\right]+\left[\frac{1}{\epsilon(k, 0)}-1\right]\right\} .
$$

We schematically indicate the resulting correction to the reaction rate in terms of an effective average value of this dynamical plasma polarization by writing

$$
\Gamma_{12}^{\text {dyn pol }}=-\beta\left\langle U_{12}^{\text {dyn pol }}\left(\mathbf{v}_{1}\right)\right\rangle \Gamma_{0} .
$$

This is essentially the result obtained by Carrero, Schäfer, and Koonin (1988) (to order $\left.e_{1} e_{2}\right)$. It corresponds to using the $e_{1} e_{2}$ term of the classical, dynamical plasma polarization energy

$$
U(t)=\frac{1}{2} \int(d \mathbf{r}) \rho(\mathbf{r}, t) \phi^{\mathrm{pol}}(\mathbf{r}, t)
$$

evaluated for

$$
\rho(\mathbf{r}, t)=e_{1} \delta\left(\mathbf{r}-\mathbf{v}_{1} t\right)+e_{2} \delta(\mathbf{r}),
$$

at $t=0$ when the two initial particles are on top of one another,

$$
U_{12}^{\mathrm{dyn} p o l}\left(\mathbf{v}_{1}\right)=e_{2} \phi_{1}^{\mathrm{pol}}(\mathbf{0}, 0)+e_{1} \phi_{2}^{\mathrm{pol}}(\mathbf{0}, 0) .
$$

This result, however, is not the whole story. The dynamical aspects of this result, which corresponds to the second-order Coulomb exchange between the two initial reacting particles as modified by the plasma, are completely cancelled by the second and third lines of Eq. (D23), which correspond to squares of first-order amplitudes that leave the plasma in excited states. These terms have exactly the same structure as the dynamical terms that have just been computed except that the time integration (D32) is now replaced by 


$$
\int_{-\infty}^{0} d t_{1} \int_{-\infty}^{0} d t_{2} e^{-i \omega^{\prime}\left(t_{1}-t_{2}\right)} e^{i \Delta E t_{1}}=\frac{1}{\Delta E}\left[-\frac{1}{\Delta E-\omega^{\prime}-i \epsilon}+\frac{1}{-\omega^{\prime}}\right]
$$

This gives rise to a correction which is the same form as that in Eq. (D33) except that the terms in the curly braces there are replaced by

$$
\left\{-\left[\frac{1}{\epsilon\left(k, \mathbf{v}_{1} \cdot \mathbf{k}\right)}-1\right]+\left[\frac{1}{\epsilon(k, 0)}-1\right]\right\} \text {. }
$$

The addition of these terms cancels the dependence upon the frequency-dependent $\epsilon\left(k, \mathbf{v}_{1} \cdot \mathbf{k}\right)$ and yields precisely the Salpeter result (D21). The moral to the story is that simple physical pictures should be augmented with a correct formal basis to ensure that correct physical results are obtained. In addition to explaining the discrepancy between our results and those of Carrero et al. (1988), this cancelation may serve as a general caveat with respect to approaches that formulate the problem completely in terms of a plasma-modified two body interaction between the fusing particles. We should note, however, that, in one circumstance, the plasma excitation terms which give rise to correction involving Eq. (D39) vanish. The frequency - $\omega$ - dependence of $\epsilon(k, \omega)$ for a classical plasma arises from a plasma particle's

velocity $\mathbf{v}_{s}$ in the form $\mathbf{v}_{s} \cdot \mathbf{k}$. Thus, if the typical velocity of the reacting particle is much less than the typical velocity of the plasma particle, $\left|\mathbf{v}_{1}\right| \ll\left|\mathbf{v}_{s}\right|$, then $\epsilon\left(k, \mathbf{v}_{1} \cdot \mathbf{k}\right)$ may be replaced by $\epsilon(k, 0)$, and the plasma excitation correction involving Eq. (D39) vanishes. This is the case when the mass of the plasma particle $M_{s}$ is much less than that of the reacting particle, $M_{s} \ll M_{1}$, as are the electrons in the plasma.

\section{APPENDIX E: DYNAMICAL CORRECTION SIZE}

Here we shall obtain the order of magnitude of the leading "dynamical" correction given in Eq. (3.30). To do this, we take the dilute plasma limit to evaluate the real-time correlation function that appears in Eq. (3.30) as

$$
\left\langle\phi(0) \phi\left(\mathbf{r}, t^{\prime}\right)\right\rangle_{\beta}=\int \frac{(d \mathbf{k})}{(2 \pi)^{3}} e^{-i \mathbf{k} \cdot \mathbf{r}}\left[\frac{4 \pi}{k^{2}}\right]^{2} \Pi^{(0)}\left(k,-t^{\prime}\right),
$$


where $\Pi^{(0)}\left(k,-t^{\prime}\right)$ is the plasma polarization function given at the end of Appendix $\mathrm{A}$ in Eq's. (A41) and (A42), but continued to real time. With both $\tau-\tau^{\prime}>0$ and $t-t^{\prime}>0$ as is appropriate to the operator ordering in Eq. (E1)], this continuation is given by $\tau-\tau \rightarrow$ $i\left(t-t^{\prime}\right)$, and we have

$$
\Pi^{(0)}\left(k,-t^{\prime}\right) \simeq \sum_{s} e_{s}^{2}\left\langle n_{s}\right\rangle_{\beta}^{(0)} C_{s}\left(k,-t^{\prime}\right)
$$

in which

$$
C_{s}\left(k,-t^{\prime}\right)=\exp \left\{i \frac{k^{2}}{2 M_{s}} t^{\prime}\right\}
$$

where we have neglected the correction in the exponential involving $t^{\prime} / \beta$ since in our application this is of the negligible order $1 / \beta Q$. The wave number integration over $\mathbf{k}$ in Eq. (E1) may be performed by using the representation

$$
\frac{1}{k^{2}}=\int_{0}^{\infty} s d s e^{-s k^{2}}
$$

interchanging integrals, and completing the square to obtain

$$
\left\langle\phi(0) \phi\left(\mathbf{r}, t^{\prime}\right)\right\rangle_{\beta}=\sum_{s} e_{s}^{2}\left\langle n_{s}\right\rangle_{\beta}^{(0)} \sqrt{4 \pi} \int_{0}^{\infty} s d s\left(s-\frac{i t^{\prime}}{2 M_{s}}\right)^{-3 / 2} \exp \left\{-\frac{\mathbf{r}^{2}}{4\left(s-i t^{\prime} / 2 M_{s}\right)}\right\} \text {. }
$$

The correction appearing in Eq. (3.30) involves the difference of this expression for $\mathbf{r} \neq 0$ and $\mathbf{r}=0$. To estimate the size of this correction, it suffices to use the classical approximation for the operator $\mathbf{r}\left(t^{\prime}\right) \simeq \mathbf{p} t^{\prime} / M$. Noting that the produced nuclei move much faster that the initial fusing nuclei, we see that the largest effect of this motion is revealed by using $p \sim \sqrt{2 M Q}$ as described in Eq. (3.20), with now $M$ a typical value of the mass of a produced nucleus. We change variables with $s=u t^{\prime} / 2 M_{s}$ to exhibit the correction as

$$
\begin{aligned}
& \pm i \beta\left(e_{1}+e_{2}\right) e_{r} \int_{0}^{t}\left\langle\phi(0)\left[\phi\left(\mathbf{r}\left(t^{\prime}\right), t^{\prime}\right)-\phi(0)\right]\right\rangle_{\beta} \\
& \sim \pm i \beta\left(e_{1}+e_{2}\right) e_{r} \sum_{s} e_{s}^{2}\left\langle n_{s}\right\rangle_{\beta}^{(0)} \sqrt{4 \pi} \int_{0}^{t} d t^{\prime} \sqrt{\frac{t^{\prime}}{2 M_{s}}} \\
& \quad \int_{0}^{\infty} u d u\left\{(u-i)^{-3 / 2} \exp \left\{-\frac{M_{s}}{M} \frac{Q t^{\prime}}{u-i}\right\}-u^{-3 / 2}\right\} .
\end{aligned}
$$


The $u$ integration is well defined, and the exponent in it involves $Q t^{\prime}$ which at most is of order unity. The overall factor of $1 / \sqrt{2 M_{s}}$ appears to imply that the light electrons in the plasma give the largest contribution. However, for the electrons, the mass ratio $m_{e} / M$ in the exponent in the $u$ integral is very small, and to leading order in this ratio, the exponential can be replaced by unity. Thus, the leading contribution from the electrons in the plasma is independent of the nature of the particle involved in the reaction, except for the overall factor of the particle's charge $e_{r}$. Since the \pm sign in Eq. (E6) is positive for the initial fusing particles 1,2 [from the $W(t)$ ] and negative for the final produced particles 3,4 [from the $V(t)$ ], and since charge is conserved, $e_{1}+e_{2}-e_{3}-e_{4}=0$, we find that the leading electron contribution vanishes. For the remaining contributions of the ions in the plasma, it suffices to replace the $u$ integral in Eq. (E6) by a (complex) constant of order unity. Since $t \sim 1 / Q$, the remaining $t$ integral is of order

$$
\int_{0}^{t} d t^{\prime} \sqrt{t^{\prime}} \sim Q^{-3 / 2}
$$

Remembering that the Debye wave number has the form $\kappa_{D}^{2}=4 \pi e^{2} \beta\langle n\rangle_{\beta}$, and that the plasma frequency of the ions $\omega_{p}$ has a size given by $\omega_{p}^{2} \sim \kappa_{D}^{2} / \beta M$, we find that the leading correction is in fact of the order

$$
i \beta\left(e_{1}+e_{2}\right) e_{r} \int_{0}^{t}\left\langle\phi(0)\left[\phi\left(\mathbf{r}\left(t^{\prime}\right), t^{\prime}\right)-\phi(0)\right]\right\rangle_{\beta} \sim \beta e_{1} e_{2} \kappa_{D}\left\{\frac{\omega_{p}}{Q} \sqrt{\frac{1}{\beta Q}}\right\} \text {. }
$$

Here we have neglected the niceties of mass and charge ratio factors, denoting all of the nuclear masses by the typical mass $M$, and replacing $\left(e_{1}+e_{2}\right) e_{r}$ by $e_{1} e_{2}$.

\footnotetext{
${ }^{14}$ The factor which replaces $Q t^{\prime}$ for the initial particles is much smaller in view of Eq. (3.19) and the discussion below it.
} 


\section{REFERENCES}

Alastuey, A. and B. Jancovici, 1978, Astrophys. J. 226, 1034.

Bahcall, J. N., 1995, Solar neutrinos: What we have learned, Physical Processes in Astrophysics, Proceedings of a Meeting in Honour of Evry Schatzman, Edited by I. W. Roxburgh, J. -L. Masnou (Springer-Verlag Berlin, Germany), pp. 19-36.

Bahcall, J. N., 1989, Neutrino Astrophysics (Cambridge Univ. Press, Cambridge, England).

Brown, L. S., 1992, Quantum Field Theory (Cambridge Univ. Press, Cambridge, England).

Carrero, C., A. Schäfer, and S.E. Koonin, 1988, Astrophys. J. 331, 565.

Clayton, D. D., 1968, Principles of Stellar Evolution and Nucleosynthesis (McGrawHill, New York).

Dar, A. and G. Shaviv, 1996, Nucl. Phys. B, Proceedings Supplements 48, 335.

Dewitt, H. E., H. C. Graboske, and M. C. Cooper, 1973, Astrophys. J. 181, 457.

Fetter, A. L. and J. D. Walecka, 1971, Quantum Theory of Many-Particle Systems, (McGraw-Hill, New York).

Gamow, G., 1928, Zeitschr. Phys. 51, 204.

Graboske, H. C., H. E. Dewitt, A. S. Grossman, and M. S. Cooper, 1973, Astrophys. J. 181, 457.

Hata, N. and P. Langacker, 1995, Phys. Rev. D 52, 420.

Ichimaru, S., 1993, Rev. Mod. Phys. 65, 255.

Jancovici, B., 1977, Journ. Stat. Phys. 17, 357. 
Johnson, C. W., E. Kolbe, S. E. Koonin, and K. Langanke, 1992, Astrophys. J. 392, 320.

Salpeter, E. E., 1954, Aust. J. Phys. 7, 373.

Salpeter, E. E. and H. M Van Horne, 1969, Astrophys. J. 155, 183.

Shaviv, N. J. and G. Shaviv, 1996, Astrophys. J. 468, 433. 